\title{
The Role of WISHE in Secondary Eyewall Formation
}

\author{
ChIEH-Jen Cheng AND CHun-CHIEH Wu \\ Department of Atmospheric Sciences, National Taiwan University, Taipei, Taiwan
}

(Manuscript received 14 August 2017, in final form 14 August 2018)

\begin{abstract}
Numerical simulations are conducted to examine the role of the wind-induced surface heat exchange (WISHE) mechanism in secondary eyewall formation (SEF). The control experiment exhibits a coherent secondary eyewall structure as quantified by various parameters (e.g., the azimuthal-mean tangential wind and vertical velocity). Prior to SEF, an area characterized by increasing diabatic heating, enhanced inertial stability, and increasing supergradient winds at the top of the boundary layer is observed outside the eyewall. While these characteristics offer the possibility of both balanced and unbalanced dynamical pathways to SEF, the focus of this study is to evaluate the impact of WISHE. To examine the sensitivity of SEF to WISHE, the surface wind used for the calculation of surface heat fluxes is capped at several designated values and at different radial intervals. When the heat fluxes are moderately suppressed around and outside the SEF region observed in the control experiment, sensitivity experiments show that the formation of the outer eyewall is delayed, and the intensity of both eyewalls is weaker. When the heat fluxes are strongly suppressed in the same region, SEF does not occur. In contrast, suppressing the surface heat fluxes in the storm's inner-core region has limited effect on the evolution of the outer eyewall. This study provides important physical insight into SEF, indicating that WISHE plays a crucial role in SEF and tropical cyclone evolution.
\end{abstract}

\section{Introduction}

Concentric eyewalls (CEs) are common features of strong tropical cyclones (TCs). Pronounced changes in the structure and intensity of TCs with CEs, typically within hours to a few days, have been documented by satellite data and aircraft observations (e.g., Houze et al. 2006, 2007; Hawkins and Helveston 2008; Kossin and Sitkowski 2009; Kuo et al. 2009; Didlake and Houze 2011; Sitkowski et al. 2011; Bell et al. 2012). Although it is well known that secondary eyewall formation (SEF) and eyewall replacement cycle (ERC) can affect the evolution of TC intensity, predicting SEF or ERC in operational models remains challenging. Furthermore, the dynamical mechanisms responsible for SEF and ERC are not well understood. It is therefore of both scientific and forecasting interest to explore the physical processes related to this phenomenon.

Numerical and observational studies have identified several mechanisms or factors related to SEF. In particular, the roles of balanced and unbalanced dynamics have

\footnotetext{
Corresponding author: Chun-Chieh Wu, cwu@typhoon.as.ntu. edu.tw
}

been evaluated in recent studies. Using the diagnostic technique developed by Eliassen (1951), Shapiro and Willoughby (1982) calculated the secondary circulation induced by point heat sources of heat and momentum in balanced, TC-like vortices and suggested that the spinup of the tangential wind, as well as the secondary circulation, can be induced by a point source of heat. From the perspective of balanced dynamics, Rozoff et al. (2012) showed that sustained latent heating outside the eyewall, which projects onto the azimuthal mean, along with enhanced inertial stability can lead to SEF. Examining the results from an idealized simulation, Wang et al. (2016) suggested that the balanced Sawyer-Eliassen solution can capture the secondary circulation of a TC with both a primary and secondary eyewall. The radial inflow is shown to spin up the tangential wind and maintain the vortex structure above the boundary layer. They also demonstrated that axisymmetric balanced dynamics alone cannot explain the spinup of the tangential wind field in the boundary layer, which results mainly from eddy processes.

Using a high-spatial- and high-temporal-resolution dataset developed in $\mathrm{Wu}$ et al. (2010, 2012), Huang et al. (2012) suggested that the unbalanced response to 
the expansion of the tangential wind field in the boundary layer serves as an important pathway for initiating and sustaining a ring of deep convection in a narrow supergradient wind zone outside the primary eyewall, leading to SEF. In addition, Wang et al. (2013) examined SEF processes from the standpoint of azimuthal-mean radial forces in the boundary layer. Their results show that the positive net radial force in the boundary layer plays a crucial role in SEF. Abarca and Montgomery (2013) showed that boundary layer dynamics alone are able to explain the development of a secondary wind maximum by using a slab boundary model forced by the tangential wind simulated in a full-physics mesoscale model. Results from both Wang et al. (2013) and Abarca and Montgomery (2013) demonstrate the important role of boundary layer processes in SEF. Different perspectives on the role of boundary layer dynamics were discussed in Kepert (2013), Kepert and Nolan (2014), and Montgomery et al. (2014).

Both observational and numerical studies have shown that an expansion of the tangential wind field is observed prior to SEF. Analysis of airborne Doppler radar and dropsonde data by Bell et al. (2012) revealed an expansion of the tangential wind field during the period of SEF (their Fig. 6). In numerical studies, it has been found that SEF occurs after the persistent expansion of the tangential wind field (e.g., Wang 2009; Wu et al. 2012; Huang et al. 2012; Wang et al. 2016). The expansion of the tangential wind field is also an integral part of other proposed mechanisms for SEF. For example, in the beta-skirt axisymmetrization process proposed by Terwey and Montgomery (2008), the expansion of the tangential wind field contributes to the formation of the vorticity skirt. As another example, Rozoff et al. (2012) suggested that SEF is triggered by increasing inertial stability outside the inner core. This increase in inertial stability is linked to the expansion of the tangential wind field.

As the wind field expands, the distribution of the surface heat fluxes also changes, affecting the structural evolution of the TC. Xu and Wang (2010) evaluated the sensitivity of the inner-core size and intensity in simulated TCs to the radial distribution of surface entropy fluxes by artificially removing the surface entropy fluxes at different radial ranges. They found that the surface entropy fluxes under the eyewall and those outside the eyewall up to about 2-3 times the radius of maximum wind are important in determining storm intensity. In addition, the surface entropy fluxes outside the eyewall play a critical role in maintaining high convective available potential energy (CAPE) and the development of spiral rainbands. The diabatic heating accompanying these rainbands drives the boundary layer inflow outside the eyewall, which spins up the TC through the radial advection of angular momentum. This advection leads to the expansion of the tangential wind field and a corresponding increase in the surface heat fluxes. Consequently, the inner-core size increases through this positive feedback.

It is well known that energy transfer from the ocean has an impact on the evolution of TCs. During the 1950s and 1960s, several studies (e.g., Riehl 1950; Kleinschmidt 1951; Ooyama 1969) pointed out the important role of surface enthalpy fluxes in TC intensification. Later, the wind-induced surface heat exchange (WISHE) mechanism was proposed by Emanuel (1986) and Rotunno and Emanuel (1987) and further discussed in Emanuel (1989, 1997). The WISHE mechanism describes the positive feedback between the sea surface heat fluxes and the surface wind speed in TC intensification. In a preexisting disturbance, surface heat fluxes strengthen the vortex, increasing the surface winds and further enhancing the heat fluxes leading to a positive feedback driving the intensification of the TC. Note that WISHE emphasizes the importance of surface heat fluxes, while convection plays a minor role in redistributing the heat (American Meteorological Society 2013). Montgomery et al. $(2009,2015)$ examined the impact of artificially suppressing WISHE in idealized experiments on TC intensification. In their experiments, surface winds were capped at a specified wind speeds (e.g., 5 and $10 \mathrm{~m} \mathrm{~s}^{-1}$ ) for the calculation of the surface heat fluxes. Their simulations showed that the storm can still strengthen with suppressed surface heat fluxes, although the intensification rate was reduced and the storm stabilized at a weaker intensity (as compared to the simulation without suppressing WISHE). Zhang and Emanuel (2016) also examined this problem using both idealized and real-world case studies. In their study on Hurricane Edouard (2014), they showed that little development occurred when the wind speed used for the calculation of the surface heat fluxes was capped at $5 \mathrm{~m} \mathrm{~s}^{-1}$, in contrast to the category- 3 hurricane seen in observations and their control experiment without suppressed surface heat fluxes. Zhang and Emanuel (2016) pointed out that real-world tropical cyclones are influenced by external factors (e.g., vertical wind shear) and that WISHE quantitatively plays an important role and may result in the difference between growth and decay of TCs.

Building on the work of these previous studies, this study focuses on how increasing surface wind and heat fluxes at the air-sea interface affects SEF. Although the impact of WISHE on intensity is well understood, the impact on SEF has not previously been investigated. The expansion of the tangential wind field is understood 
to be a key component of the SEF process. The WISHE mechanism will, therefore, clearly be important in the expansion, since it describes the positive feedback between the surface wind speed and surface energy fluxes. As the tangential wind field expands and intensifies, the surface heat fluxes will also increase in the area where the secondary eyewall forms gradually. It is, therefore, important to understand the potential role of WISHE in SEF. This work is focused on examining the sensitivity of SEF to the surface heat fluxes and the surface wind speed at different radial intervals.

This study evaluates the role of WISHE in both the inner and outer eyewalls in SEF. The numerical experiments are divided into two groups: a control experiment (CTL) and several sensitivity experiments designed to evaluate the role of WISHE in SEF. Section 2 of this paper describes the modeling framework used in this study. In section 3 , the evolution and features of CTL are discussed. Section 4 presents the detailed analyses of the sensitivity experiments. Finally, the discussion and conclusions are presented in section 5 .

\section{Model and experiment designs}

\section{a. $C T L$}

The Advanced Research Weather Research and Forecasting (WRF) Model is used in this study to conduct simulations of Typhoon Sinlaku (2008), with the vortex generated based on the ensemble Kalman filter (EnKF) data assimilation method as described in Wu et al. (2012), but with higher horizontal resolution. The sizes of the four domains are $91 \times 82(\mathrm{~d} 1), 85 \times 85(\mathrm{~d} 2), 115 \times 115$ (d3), and $190 \times 190(\mathrm{~d} 4)$, with grid spacings of $45,15,5$, and $1.67 \mathrm{~km}$, respectively. The initial vortex is generated at 1800 UTC 4 September 2008, and the data assimilation method is applied to each domain until 1500 UTC 10 September 2008. The vortex used in CTL is taken from the numerical integration at 1800 UTC 10 September 2008, which is defined as $0 \mathrm{~h}$ of the CTL simulation. A 54-h simulation is carried out from 1800 UTC 10 September $(0 \mathrm{~h})$ to 0000 UTC 13 September 2008 (54 h). The track of the typhoon is different from the real storm in that the simulated vortex moves slowly northward without approaching the terrain of Taiwan (not shown). As a result, the vortex is only slightly influenced by the terrain, and the main structure of the vortex remains over open ocean.

There are 35 eta levels with more condensed levels in the boundary layer. The physics parameterization schemes used in this study include the WSM6 microphysics scheme (Hong and Lim 2006), the Rapid Radiative
Transfer Model (RRTM) scheme for longwave radiation (Mlawer et al. 1997), the Dudhia scheme (Dudhia 1989) for shortwave radiation, the Yonsei University (YSU) planetary boundary layer scheme (Hong et al. 2006), and the Kain-Fritsch cumulus scheme (Kain and Fritsch 1993) on the two coarse domains. The sea surface temperature is set at a fixed value of $29^{\circ} \mathrm{C}$ over the whole domain.

\section{b. Sensitivity experiments}

To examine the sensitivity of SEF to surface heat fluxes (referred to as WISHE) in different radial intervals of a TC (such as in the storm core or the outer region), the surface wind is capped at several designated values in the calculation of total surface heat fluxes. The formulas for surface heat fluxes (from Jiménez et al. 2012) are shown below:

$$
\begin{aligned}
& \mathrm{SH}=-\rho c_{p} C_{h}(\Delta \theta)\left[\min \left(U_{\max }, U\right)\right] \text { and } \\
& \mathrm{LH}=-\rho L_{v} M C_{q}(\Delta q)\left[\min \left(U_{\max }, U\right)\right],
\end{aligned}
$$

where $\mathrm{SH}$ and $\mathrm{LH}$ are sensible and latent heat fluxes, respectively; $\rho$ is the air density; $c_{p}$ is the specific heat capacity; $C_{h}$ and $C_{q}$ are the dimensionless bulk transfer coefficients; $L_{v}$ is the latent heat of vaporization; and $M$ is the surface moisture availability (constant value 1 over the ocean). In addition, $\Delta \theta$ and $\Delta q$ are the differences of potential temperature and saturated specific humidity between the sea surface and the surface layer of the atmosphere. To suppress WISHE, the surface wind speed is taken to be $\min \left(U_{\max }, U\right)$, the minimum of the true wind speed in the surface layer $U$ and the specified capped wind speed $U_{\max }$ (see the values below) in the calculation of the surface heat fluxes. The values of $U_{\max }$ are set at $15,10,5$, and $1 \mathrm{~m} \mathrm{~s}^{-1}$. This method of suppressing WISHE is the same as that used in related works (e.g., Montgomery et al. 2009; Zhang and Emanuel 2016). With only one variable changed to suppress WISHE, this method provides a simple way to examine the role of WISHE in SEF.

The regions in which the surface heat fluxes are suppressed are defined as the OSC (outside the small circle; $r>50 \mathrm{~km}$ ), OBC (outside the big circle; $r>80 \mathrm{~km}$ ), InC (inner circle; $r<50 \mathrm{~km}$ ), and $\mathrm{RiR}$ (ring region; $55<r<$ $90 \mathrm{~km}$ ), respectively (Fig. 1). From the evolution of vertical velocity and tangential winds observed in CTL (Fig. 2a), the majority of the convection associated with the primary eyewall is within a radius of $50 \mathrm{~km}$ from the center. The OSC and InC experiments can, therefore, help to evaluate the role of WISHE within or outside the radius of the eyewall convection. In addition, the results 

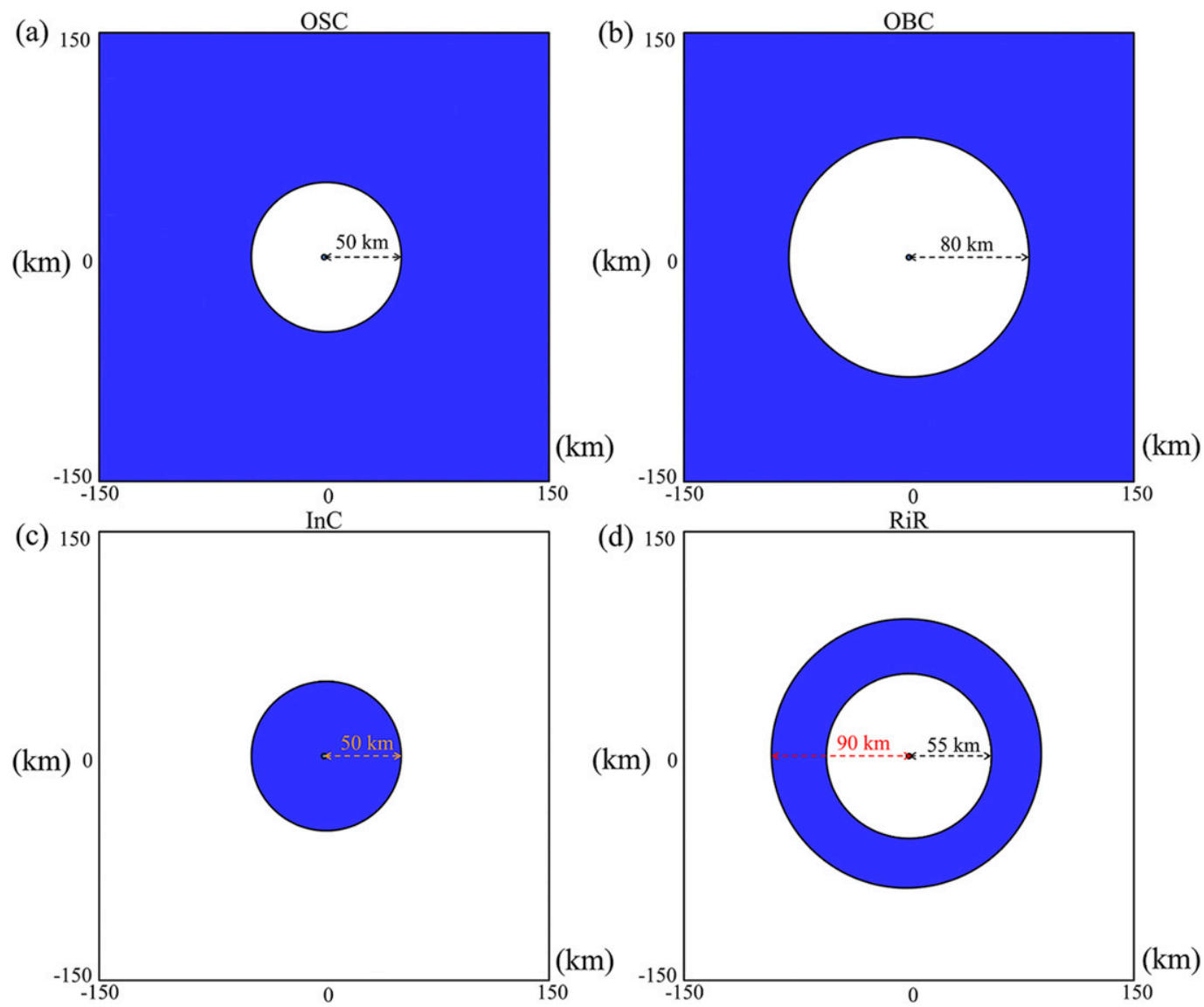

FIG. 1. Experiment designs of the sensitivity experiments. The blue area indicates the region in which WISHE is suppressed in (a) OSC, (b) OBC, (c) InC, and (d) RiR.

of the OBC experiments are compared with those of the OSC experiments in order to evaluate the sensitivity of TC structure to surface heat fluxes outside the primary eyewall. In the RiR experiments, the suppressed region is set at a radius of $55-90 \mathrm{~km}$, since this is the radius in which most of the convection associated with the secondary eyewall is contained in CTL. In total, 16 sensitivity experiments with suppressed surface heat fluxes are conducted. Each sensitivity experiment with suppressed WISHE begins at $t=12 \mathrm{~h}$ using restart files from CTL. In all experiments, the time of SEF is defined as when a persistent secondary maximum of azimuthally averaged tangential wind at $1-\mathrm{km}$ height is observed.

\section{Results-CTL}

\section{a. An overview of the CTL simulation}

Figure 2a presents a time-radius diagram of the azimuthal-mean tangential wind and vertical velocity in CTL. Both parameters clearly demonstrate the secondary eyewall structure at the time of SEF, that is, at $31.5 \mathrm{~h}$. Before $36 \mathrm{~h}$, the inner eyewall is located between the 20 - and $40-\mathrm{km}$ radii (the area with high vertical velocity). After $24 \mathrm{~h}$, convection begins to form outside the $60-\mathrm{km}$ radius, along with a significant broadening of the axisymmetric tangential wind field. The convection in the outer region becomes more organized, and the tangential winds significantly expand after $30 \mathrm{~h}$, with a concentric eyewall structure visible at this stage. After $39 \mathrm{~h}$, the inner eyewall diminishes, while the updraft and the tangential winds in the outer eyewall intensify. Figure $2 \mathrm{~b}$ shows a time-radius diagram of the distribution of the CAPE and vertical velocity. The CAPE is greater than $1400 \mathrm{~J} \mathrm{~kg}^{-1}$ outside the primary eyewall region at $12 \mathrm{~h}$. This large CAPE value shows that the region outside the primary eyewall is thermodynamically favorable for the development of convection prior to SEF. CAPE is consumed after the formation of active convection $\left(w>0.5 \mathrm{~m} \mathrm{~s}^{-1}\right)$ around $28 \mathrm{~h}$, and is continually consumed after SEF. These features are consistent with previous studies (e.g., Wang et al. 2016). 
(a)

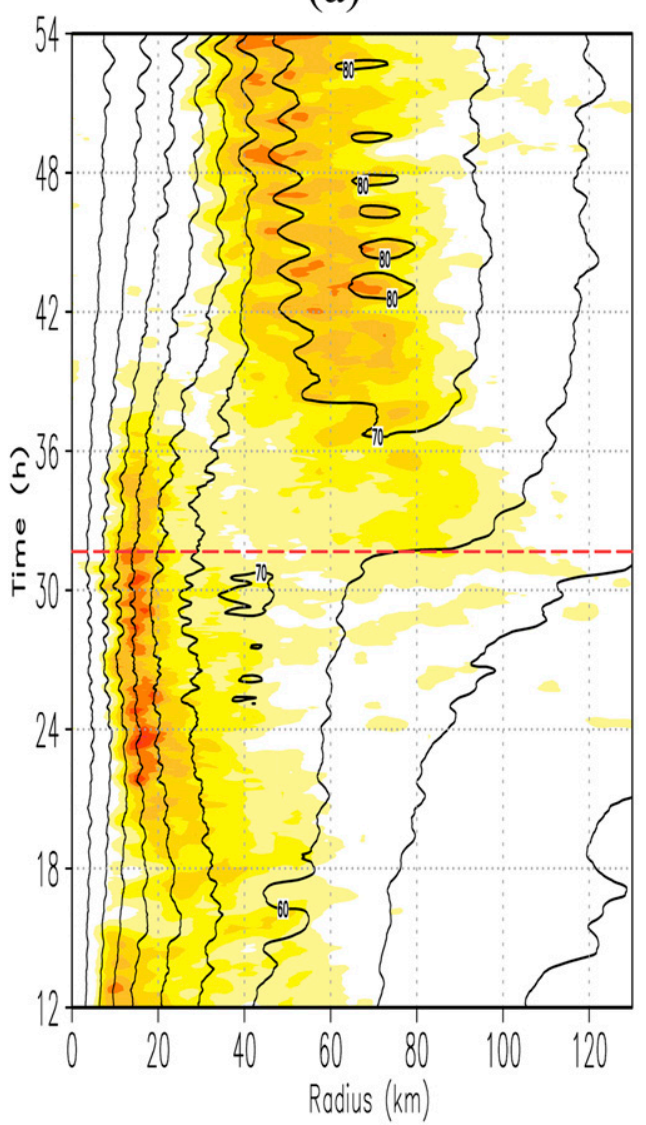

$\begin{array}{llllllllll}0.2 & 0.4 & 0.6 & 0.8 & 1 & 1.2 & 1.4 & 1.6 & 2 & 2.4\end{array}$ (b)

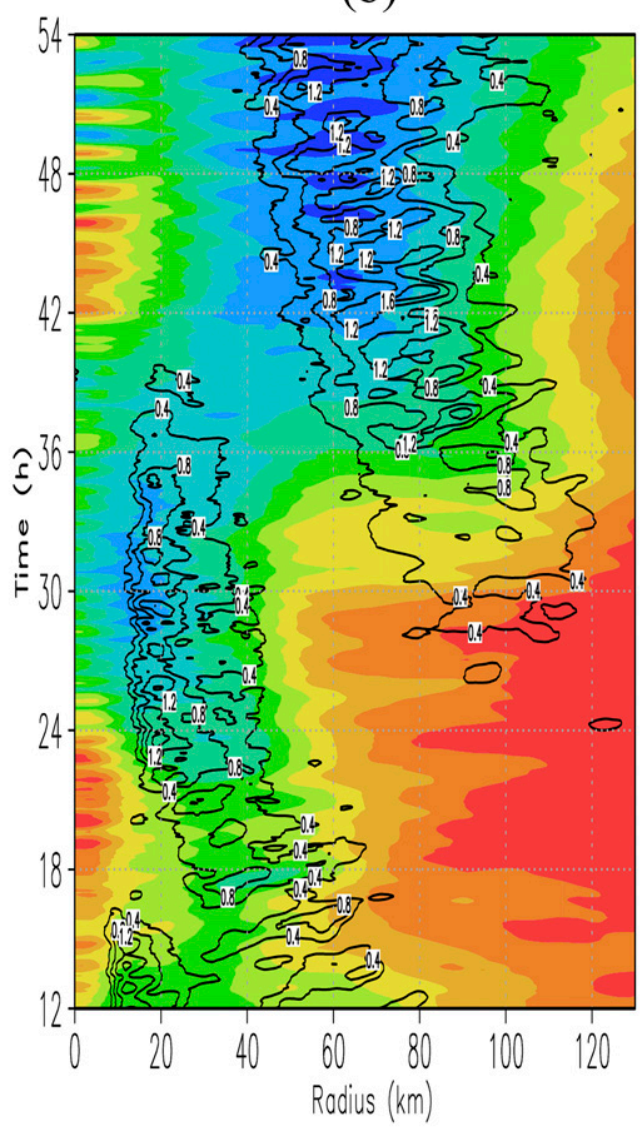

$\begin{array}{lllllllll}400 & 600 & 800 & 1000 & 1200 & 1400 & 1600 & 1800 & 2000\end{array}$

FIG. 2. (a) Time-radius Hovmöller diagram of the azimuthal-mean vertical velocity (shaded; $\mathrm{m} \mathrm{s}^{-1}$ ) and $1-\mathrm{km}$ tangential wind (contours; $\mathrm{m} \mathrm{s}^{-1}$ ). The red dashed line indicates the time of SEF. (b) Time-radius Hovmöller diagram of the azimuthal-mean vertical velocity at $3-\mathrm{km}$ altitude (contours for values $\geq 0.4 \mathrm{~m} \mathrm{~s}^{-1}$ ) and CAPE (shaded; $\mathrm{J} \mathrm{kg}^{-1}$ ).

\section{b. Dynamical features of the CTL simulation}

Figure 3a shows a time-radius diagram of the azimuthalmean tangential wind at $1-\mathrm{km}$ altitude and diabatic heating rate vertically averaged between 1 - and $10-\mathrm{km}$ altitudes. A secondary peak in the diabatic heating appears at around $27 \mathrm{~h}$ (Fig. 3a), which is associated with active convective activity (Fig. 2b) and the area of expanding tangential winds outside the primary eyewall (Fig. 3a). Figures $3 b$ and $3 c$ show the changes in inertial stability $I^{2}$ and potential vorticity (PV) from 22 to $32 \mathrm{~h}$, which is the time period from before to immediately after SEF. Inertial stability is calculated by

$$
I^{2}=\left(\bar{f}+\frac{\bar{v}}{r}\right)(\bar{f}+\bar{\zeta})
$$

where $\bar{f}$ is the Coriolis parameter, $\mathrm{r}$ is the radius, $\bar{v}$ is the tangential wind, and $\bar{\zeta}$ is the relative vorticity in the $z$ direction. The overbar denotes an azimuthal-mean quantity. Figures $3 \mathrm{~b}$ and $3 \mathrm{c}$ show that $I^{2}$ and PV both increase in the outer region $(60-120 \mathrm{~km})$, since the secondary circulation induced by the convection can lead to the expansion of the tangential wind field and the increase of $I^{2}$. The increase in PV can also be explained by the active convective heating in this region. In addition, the increase in $I^{2}$, which is accompanied by latent heating (around the radius of $60-120 \mathrm{~km}$ ), further facilitates heating efficiency in enhancing the tangential winds (e.g., Schubert and Hack 1982; Shapiro and Willoughby 1982). These features are in good agreement with the findings shown in Rozoff et al. (2012) and other related works.

The gradient wind balance relationship, agradient wind $\overline{v_{\mathrm{ag}}}$, and agradient force ( $\overline{\mathrm{AF}}$; sum of the azimuthalmean radial pressure gradient force and the Coriolis and centrifugal forces) are 
(a)

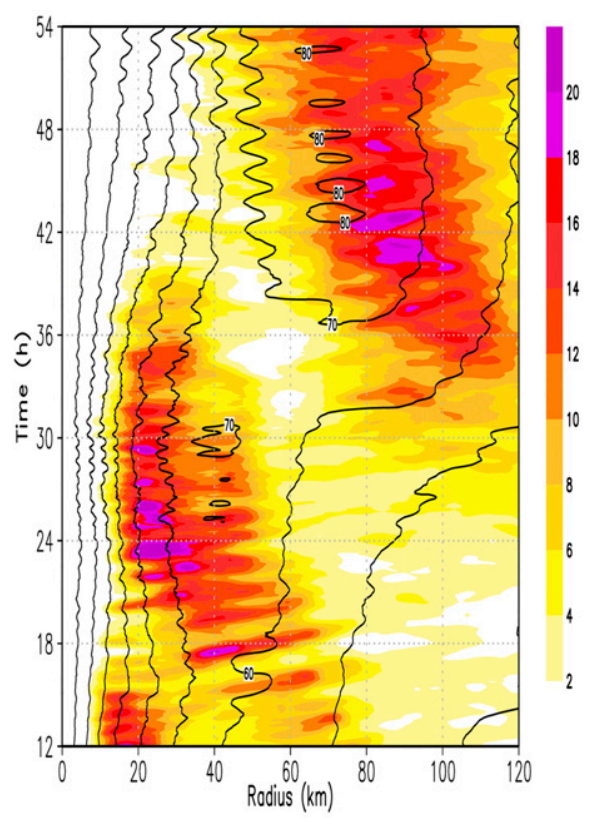

(b)

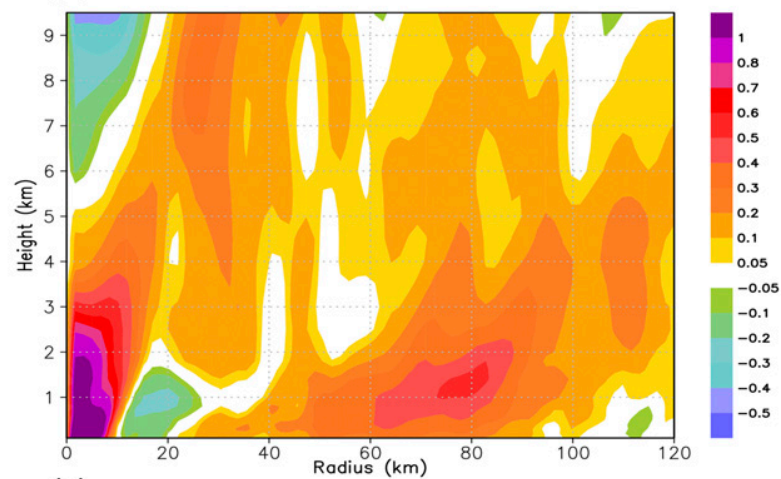

(c)

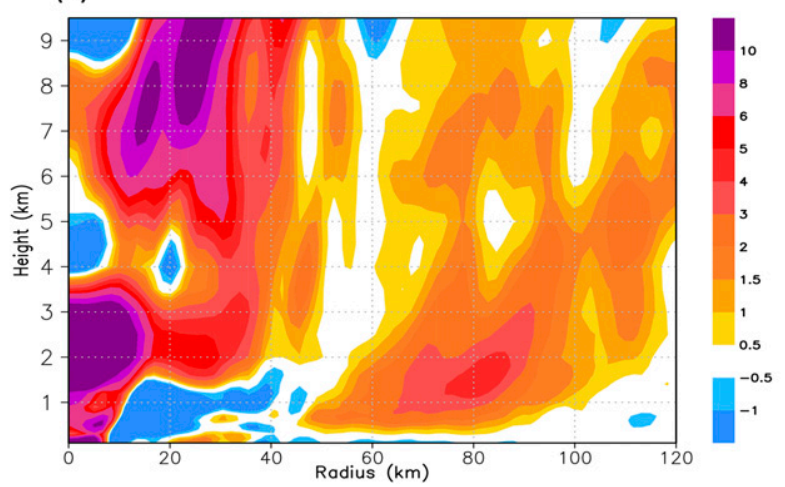

FIG. 3. (a) Time-radius Hovmöller diagram of the 1-km tangential wind (contours; $\mathrm{m} \mathrm{s}^{-1}$ ) and vertically averaged diabatic heating (shaded; $\mathrm{K} \mathrm{h}^{-1}$ ). (b),(c) Radius-height cross sections of the changes (between 22 and $32 \mathrm{~h}$ ) in the azimuthally averaged inertial stability $\left(\mathrm{s}^{-1}\right)$ and PV (PVU; $1 \mathrm{PVU}=10^{-6} \mathrm{~K} \mathrm{~kg}^{-1} \mathrm{~m}^{2} \mathrm{~s}^{-1}$ ), respectively.

$$
\begin{aligned}
\frac{1}{\rho} \frac{\partial \bar{p}}{\partial r} & =\left(f \overline{v_{g}}+\frac{\bar{v}_{g}^{2}}{r}\right), \\
\overline{v_{\mathrm{ag}}} & =\bar{v}-\overline{v_{g}}, \quad \text { and } \\
\overline{\mathrm{AF}} & =-\frac{1}{\rho} \frac{\partial \bar{p}}{\partial r}+\left(f \bar{v}+\frac{\bar{v}^{2}}{r}\right),
\end{aligned}
$$

where $\overline{v_{g}}$ is the gradient wind and $p$ is pressure. Once the boundary layer tangential winds become supergradient $\left(\overline{v_{\mathrm{ag}}}>0\right.$; that is to say, $\overline{\mathrm{AF}}>0$, the net force between the pressure gradient force, Coriolis force, and centrifugal force is directed radially outward), the boundary layer inflow rapidly decelerates. This deceleration leads an area of boundary layer convergence, favorable for air to erupt out of the boundary layer. Figure 4 shows radius-height diagrams of variables in the lower troposphere from 20 to $40 \mathrm{~h}$, indicating the flow evolution prior to, and after, SEF. First, the inflow below $1 \mathrm{~km}$ strengthens and expands outward. The strengthening inflow leads to the broadening of the tangential wind field in the boundary layer, since larger absolute angular momentum $M$ is advected inward by the strong inflow below $1.5 \mathrm{~km}$. Additional analyses (not shown) show that axisymmetric flow dominates the spinup of the tangential winds; however, the detailed processes governing this process are beyond the scope of this study. Second, the supergradient winds near the top and above the boundary layer $(0.3<z<2.5 \mathrm{~km})$ increase with time at radii between 50 and $100 \mathrm{~km}$. Third, the boundary layer inflow decreases with decreasing radius inside the radial interval between 50 and $100 \mathrm{~km}$ at around $31.5 \mathrm{~h}$, implying the presence of a convergence zone. Fourth, the region with maximum outflow at the top of the boundary layer moves from the inner eyewall to the outer eyewall from 20 to $40 \mathrm{~h}$. In all, the results are generally consistent with findings in Huang et al. (2012) that the unbalanced boundary layer response to an expanding tangential wind field can play a role in concentrating and sustaining deep convection in a supergradient wind zone in the outer region, serving as a progressive pathway to SEF. 


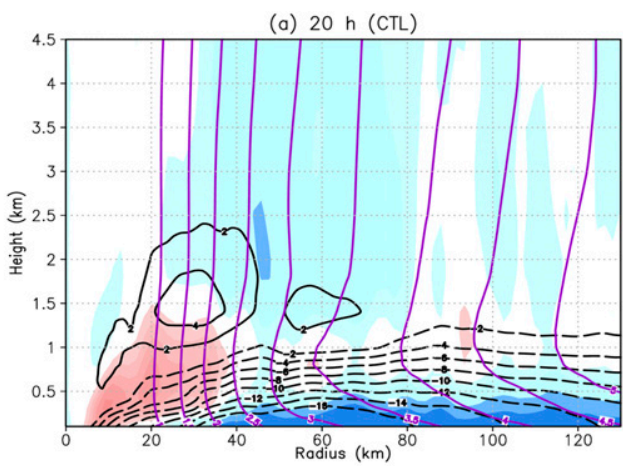

(c) $30 \mathrm{~h}$ (CTL)

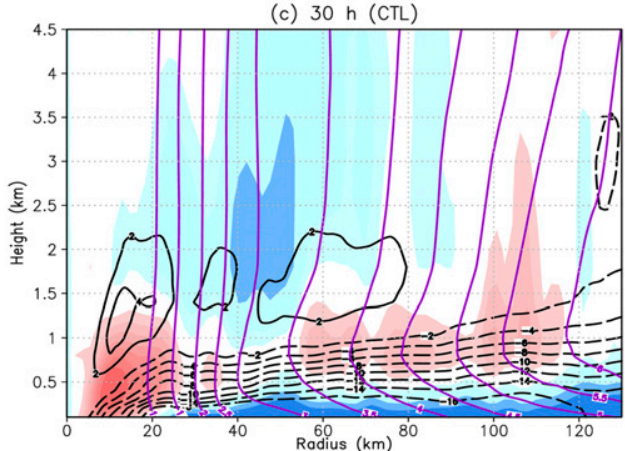

(e) $35 \mathrm{~h}$ (CTL)

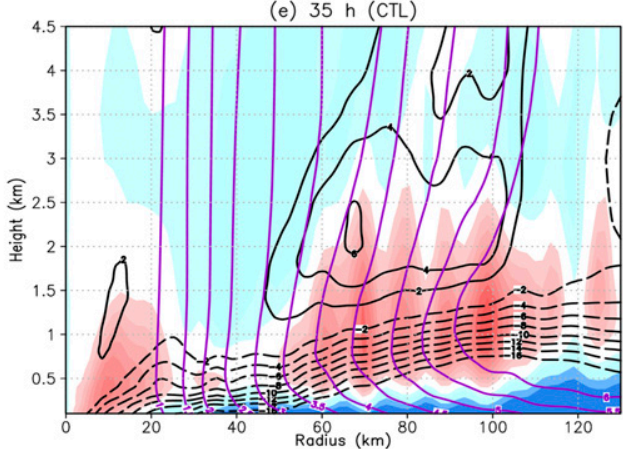

(b) $25 \mathrm{~h}(\mathrm{CTL})$

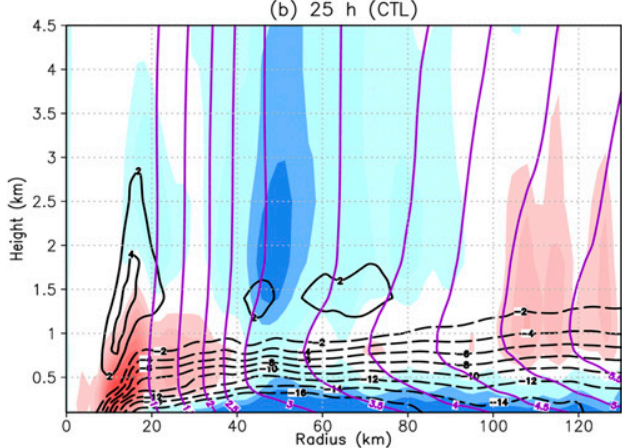

(d) $31.5 \mathrm{~h}$ (CTL)

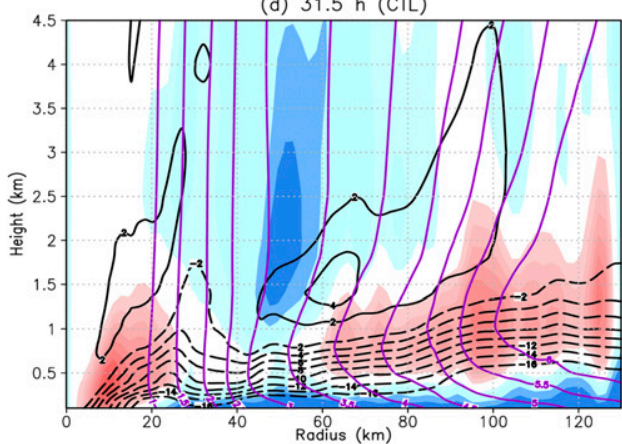

(f) $40 \mathrm{~h}$ (CTL)

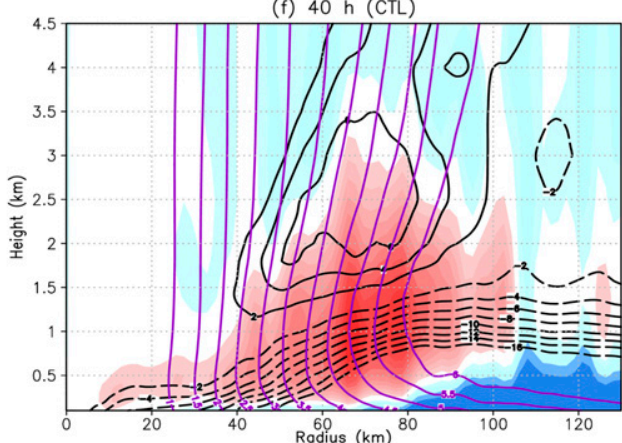

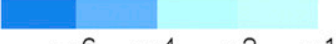

$\begin{array}{llll}-6 & -4 & -2 & -1\end{array}$

$\begin{array}{llll}1 & 2 & 3 & 4\end{array}$

5

6

7

1

1112

FIG. 4. Radius-height diagrams of the subgradient wind (blue shaded; $\mathrm{m} \mathrm{s}^{-1}$ ), supergradient wind (red shaded; $\mathrm{m} \mathrm{s}^{-1}$ ), radial inflow (black dashed lines; $\mathrm{m} \mathrm{s}^{-1}$ ), and radial outflow (black solid lines; $\mathrm{m} \mathrm{s}^{-1}$ ) from (a) 20 to (f) $40 \mathrm{~h}$. The purple contours indicate the azimuthally averaged absolute angular momentum surfaces $\left(10^{6} \mathrm{~m}^{2} \mathrm{~s}^{-1}\right)$.

\section{Results-Sensitivity experiments}

\section{a. An overview of the experiments}

Figure 5 shows the evolution of the maximum 10-m wind speed in all experiments. In the OSC (Fig. 5a) and OBC (Fig. 5b) experiments, the storms are mostly weaker than CTL (black line) throughout the integration period. In the InC (Fig. 5c) and the RiR (Fig. 5d) experiments, although the intensity of the TCs is weaker than in CTL before $35 \mathrm{~h}$, their final intensities are close in most cases. The radial distribution of the azimuthally averaged surface heat fluxes of each experiment is shown in Fig. 6, which is averaged from 13 to $18 \mathrm{~h}$. The location of the reduced heat fluxes coincides well with the area of suppressed WISHE.

Figures 7 and 8 show time-radius diagrams of the azimuthal-mean tangential wind and vertical velocity in the OSC and OBC experiments, respectively. SEF is not observed in OSC-01, OSC-05, OBC-01, and OBC-05, and the intensity of these storms weakens gradually. In experiments in which the surface fluxes are capped to a lesser degree (OSC-10, OSC-15, OBC-10, and OBC-15), SEF still occurs, but its onset time is delayed for a few hours ( $7 \mathrm{~h}$ for OSC-10 and $1 \mathrm{~h}$ for 
(a)

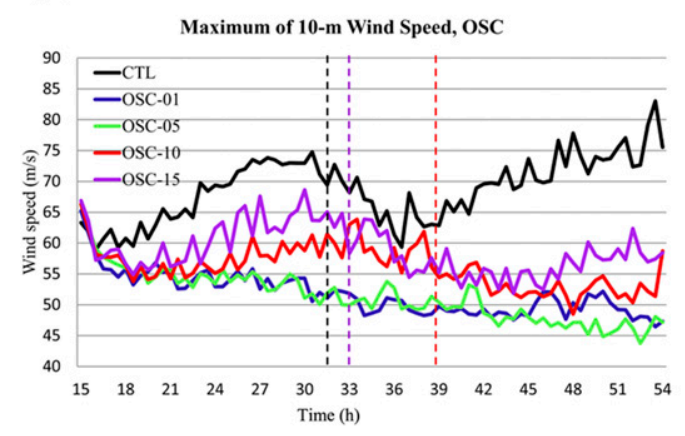

(c)

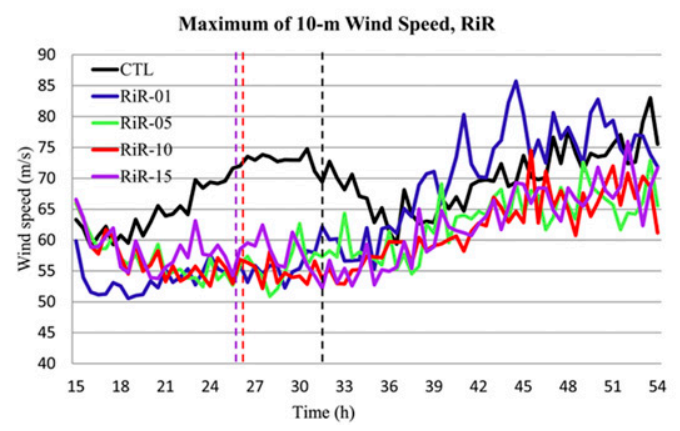

(b)

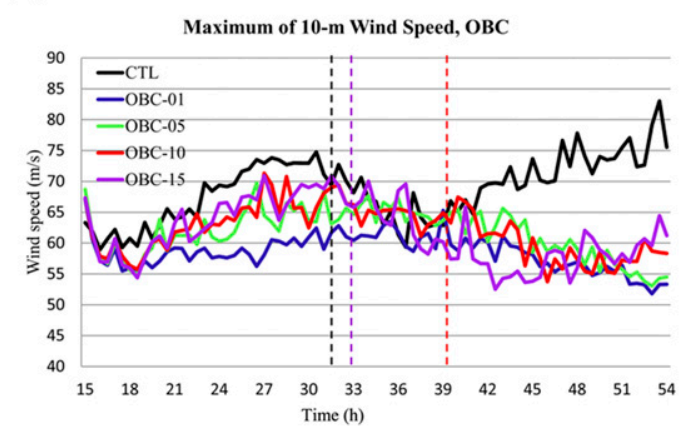

(d)

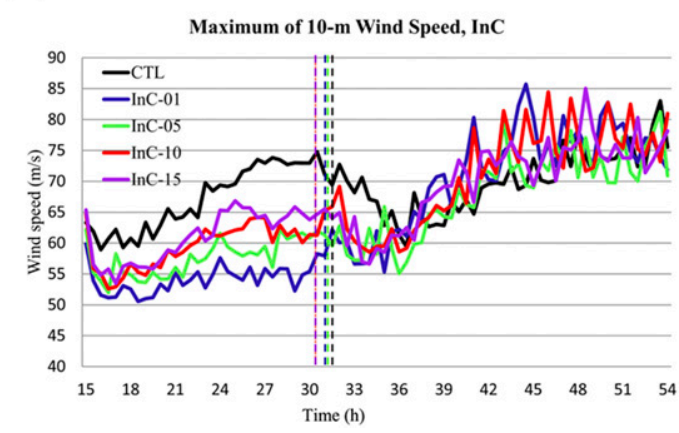

FIG. 5. The maximum 10-m wind speed $\left(\mathrm{m} \mathrm{s}^{-1}\right.$ ) of each experiment: (a) OSC, (b) OBC, (c) RiR, and (d) InC. The dashed line indicates the time of SEF. In (d), the dashed lines indicate the SEF time of second episode of SEF.

OSC-15) as compared to CTL. Moreover, the intensity of both eyewalls in these cases is weaker than CTL, both in terms of winds and convection (Fig. 5 and cf. Fig. 2, Fig. 6, and Fig. 7). In all, the WISHE feedback outside the primary eyewall impacts both the intensity of the primary and secondary eyewalls and the time of SEF. When the surface fluxes are strongly suppressed, SEF does not occur, suggesting the crucial role of WISHE in SEF.

To further investigate the relative role of WISHE in different regions, the surface heat fluxes are capped in the ring area (RiR experiments; Fig. 9) where the secondary eyewall forms in CTL. In all RiR experiments, convection around the $20-\mathrm{km}$ radius weakens gradually, while a wide area of convection develops at radii between 40 and $60 \mathrm{~km}$, which is defined as an SEF episode. The moat region (at radii between 25 and $40 \mathrm{~km}$ ) in the early SEF is caused by capping the surface heat fluxes in the ring region, since this moat region is not found in CTL. Then, in RiR-01 and RiR-05, convection at radii between 40 and $60 \mathrm{~km}$ replaces the original eyewall. After this, the new eyewall intensifies and continues to expand with time. On the other hand, in RiR-10 and RiR-15, except for the early formation of a secondary peak of convection, another episode of SEF can be identified with a secondary peak of tangential wind around $26 \mathrm{~h}$. During the second episode of SEF, a moat region can be found in RiR-15 (ranging from 30- to $50-\mathrm{km}$ radii), but is not clear in RiR-10. This may be related to the weak primary eyewall that is caused by less surface heat fluxes fed into this area, while having no contraction after the first SEF. In addition, the secondary peak of the tangential wind in RiR-10 in the second episode is not well recognized, but other parameters (which are shown in the following part) can indicate the existence of the secondary eyewall. In all, the RiR experiments indicate that the primary eyewall is sensitive to the surface heat fluxes in the ring region just outside the inner eyewall. It is likely that suppression of the surface heat fluxes around the ring region would reduce the heat energy entering the primary eyewall since the boundary layer inflow would pass over the heat-flux-reduced region. This will be discussed later in section $4 \mathrm{~b}$. Furthermore, the surface heat fluxes of the ring region may affect location of the secondary eyewall. The secondary eyewalls in all the RiR experiments expand with time, with the final radius of the secondary eyewall farther from the center than that of CTL, which can be shown by the area with strong convection. 
(a)

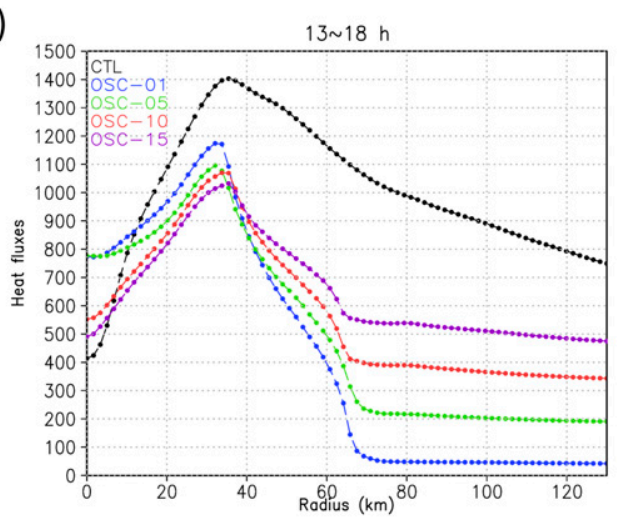

(c)

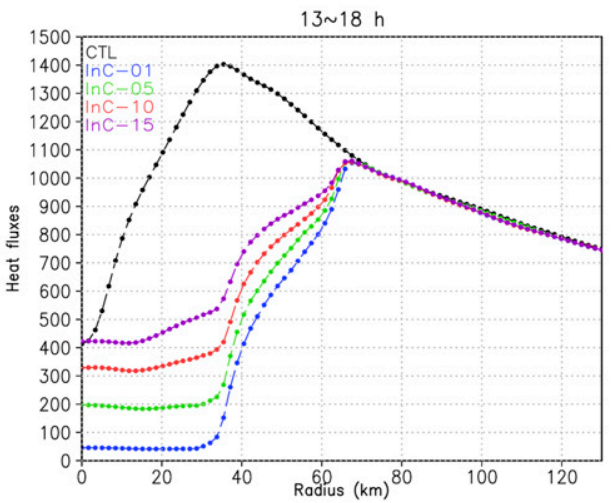

(b)

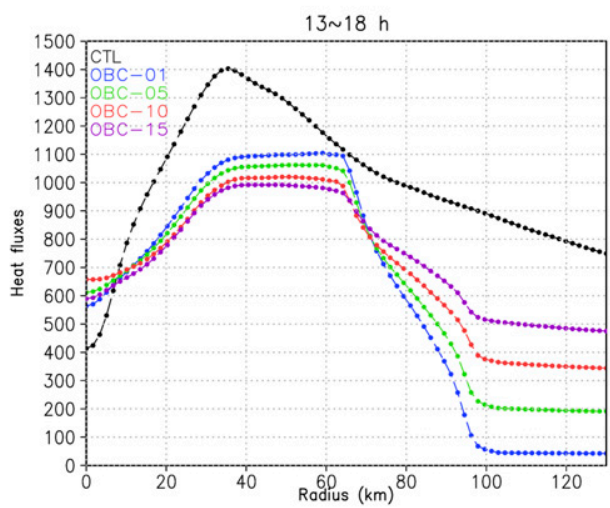

(d)

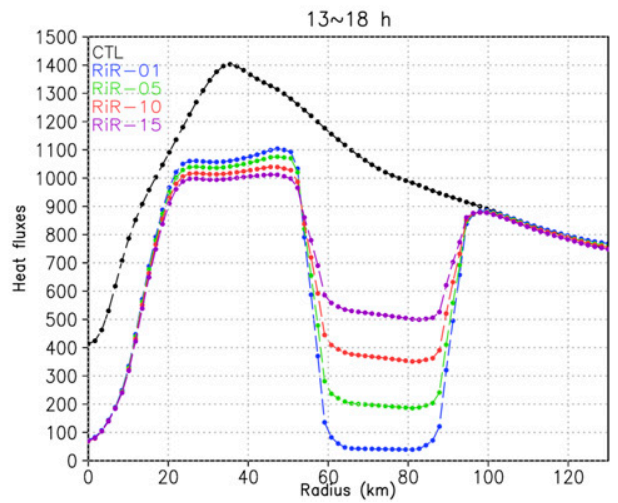

FIG. 6. Radial distribution of the azimuthal-mean surface heat fluxes $\left(\mathrm{W} \mathrm{m}^{-2}\right)$, averaged from 13 to $18 \mathrm{~h}$ in (a) OSC, (b) OBC, (c) InC, and (d) RiR. Each color refers to a different specified capped wind speed. Blue, green, red, and purple indicate $1,5,10$, and $15 \mathrm{~m} \mathrm{~s}^{-1}$, respectively.

Figure 10 shows the results from the InC experiments. SEF occurs in all InC experiments with an outer eyewall as strong as that in CTL. The results of the InC experiments demonstrate WISHE around the inner-core region is not a crucial factor for SEF; however, suppressing the surface heat fluxes can result in a shorter-lived inner eyewall.

\section{b. Dynamical characteristics of sensitivity experiments}

Figure 11 shows time-radius diagrams of azimuthalmean CAPE and vertical velocity for six experiments: OSC-05, OSC-10, OSC-15, InC-05, RiR-10, and RiR-15. In each experiment, the magnitude of the CAPE in each capped area decreases because of the suppression of WISHE. With reduced CAPE, the convection of the secondary eyewall is weaker in OSC-10 (Fig. 11b) and OSC-15 (Fig. 11c). In addition, in InC-05 (Fig. 11d), capping the surface heat fluxes inside the $50-\mathrm{km}$ radius causes a decrease in CAPE from the start of the simulation, as compared to the results of CTL (Fig. 2b). This results in a weaker and shorter-lived inner eyewall since there is less energy (CAPE) to support the deep convection in this region. Moreover, suppression of the WISHE feedback in the ring region leads to the reduction of CAPE around and inside the suppressed region (20-80-km radii) in RiR-10 and RiR-15 (Figs. 11e,f), as compared to the CAPE in CTL (Fig. 2b). This reduction demonstrates that less heat energy is transported inside the ring region by the inflow, which passes over the area of suppressed heat fluxes. Convection is weaker inside the ring region in both RiR-10 and RiR-15 (cf. Figs. 9c,d and Figs. 11e,f).

From the viewpoint of balanced dynamics (e.g., Rozoff et al. 2012), the distribution of diabatic heating and the change in the inertial stability are both important for SEF. Figure 12 shows the vertical distribution of the temporally and azimuthally averaged diabatic heating. In the OSC experiments (Figs. 12a-c), weaker diabatic heating is observed in the region where WISHE is suppressed. This reduced diabatic heating rate becomes increasingly evident when WISHE is suppressed to a greater extent (OSC-05). SEF is observed in InC-05, with a clear secondary peak of diabatic heating. In RiR-10 and RiR-15, capping heat fluxes in the ring region greatly affects the distribution of the diabatic heating, 
(a) 0 SC-01

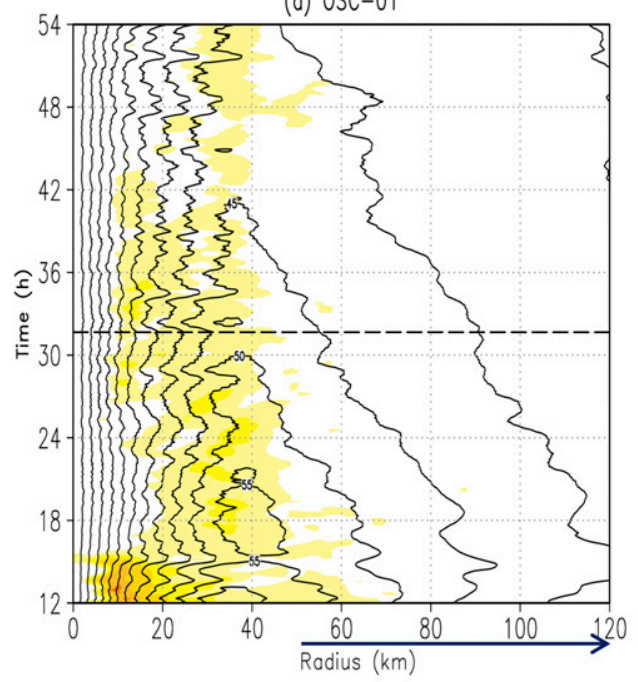

(c) OSC-10

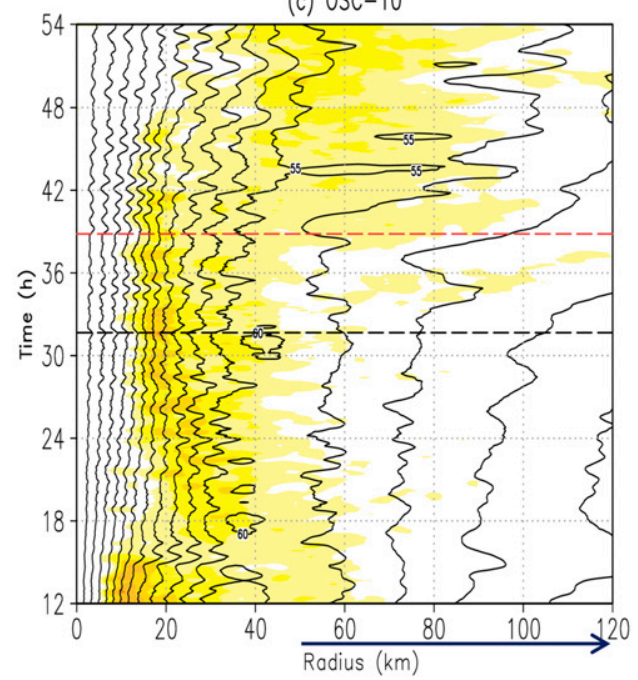

(b) $0 \mathrm{SC}-05$

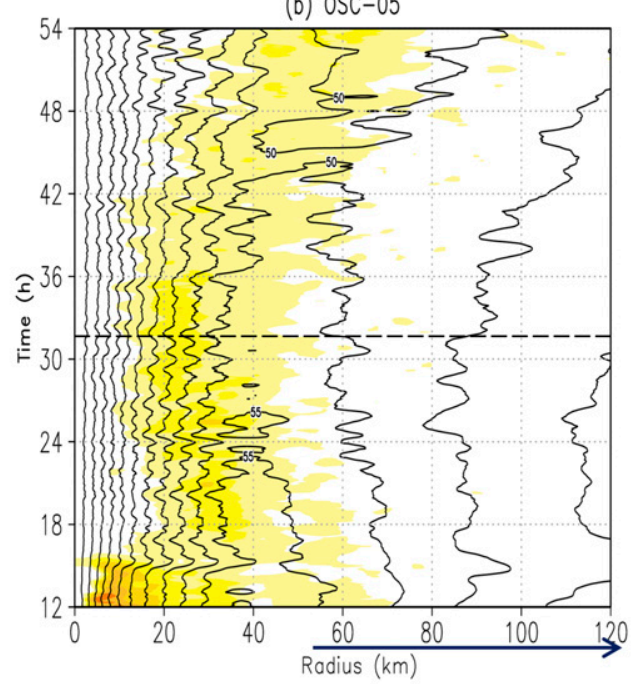

(d) OSC-15

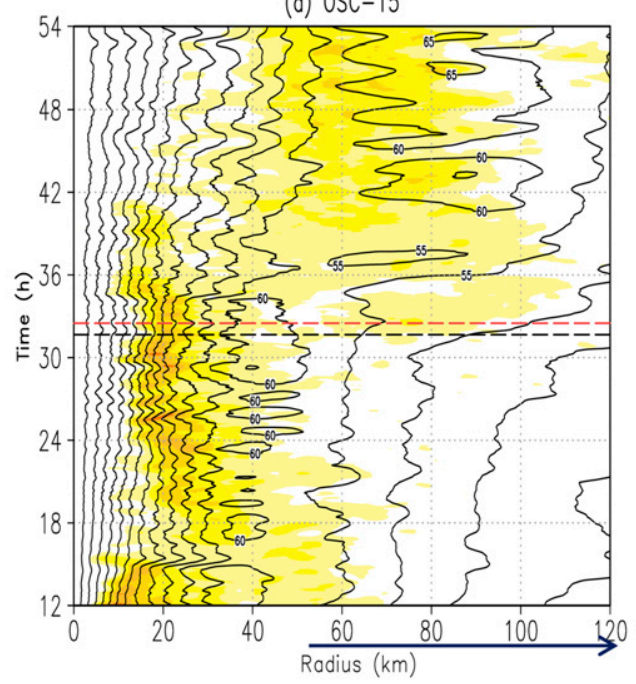

\section{$\begin{array}{llllllllll}0.2 & 0.4 & 0.6 & 0.8 & 1 & 1.2 & 1.4 & 1.6 & 2 & 2.4\end{array}$}

FIG. 7. Time-radius Hovmöller diagrams of the azimuthal-mean vertical velocity (shaded; $\mathrm{m} \mathrm{s}^{-1}$ ) and $1-\mathrm{km}^{-}$ tangential wind (contours; $\mathrm{m} \mathrm{s}^{-1}$ ) of (a) OSC-01, (b) OSC-05, (c) OSC-10, and (d) OSC-15. The black dashed line indicates the SEF time in CTL, and the red dashed line indicates the SEF time in each experiment. The blue arrows indicate the selected regions of suppressed heat fluxes.

especially in the primary eyewall region. In RiR-10, there are three peaks of the diabatic heating. The innermost and weakest one is the primary eyewall before the first episode of SEF. The other two are caused by the expanding eyewall (cf. Fig. 9c, new eyewall after first episode of SEF) and the outer eyewall of the second episode of SEF. In RiR-15, two separated peaks are found, which are associated with the inner and outer eyewalls. In addition, while the surface heat fluxes are suppressed around the secondary peak of diabatic heating, the residual surface heat fluxes are adequate for developing a secondary peak of diabatic heating and the secondary eyewall. Furthermore, the secondary eyewall, which gradually expands with time, can also be supported by the energy from larger radii.

Changes in the inertial stability before the time of SEF in each experiment are shown in Fig. 13. In OSC-10, OSC-15, InC-05, and RiR-15, the inertial stability increases in the SEF region (at radii between 50 and $120 \mathrm{~km}$ ), while suppression of WISHE in the outer region (OSC-10 and OSC-15) leads to smaller increases (cf. with CTL; Fig. 3b) in inertial stability. This smaller 


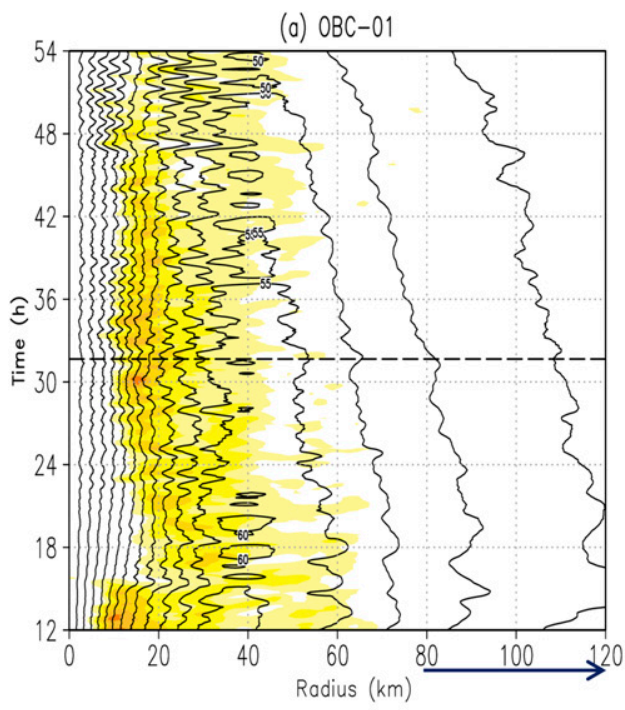

(c) $\mathrm{OBC}-10$

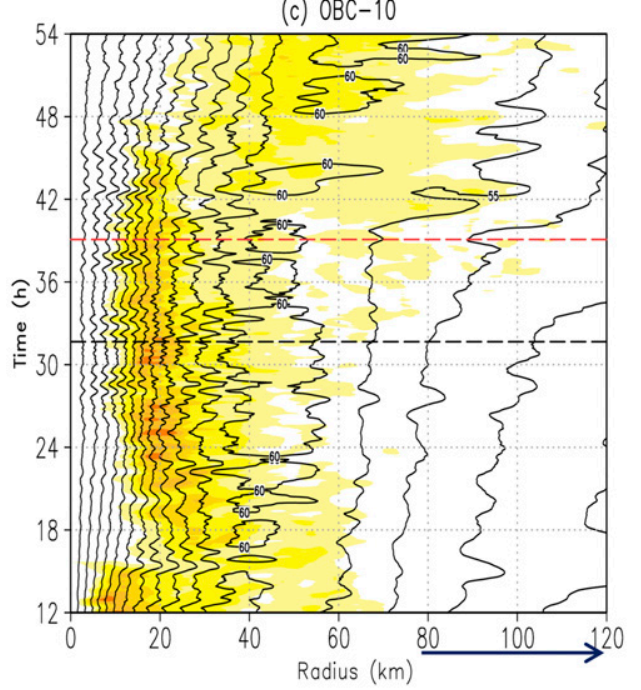

(b) $\mathrm{OBC}-05$

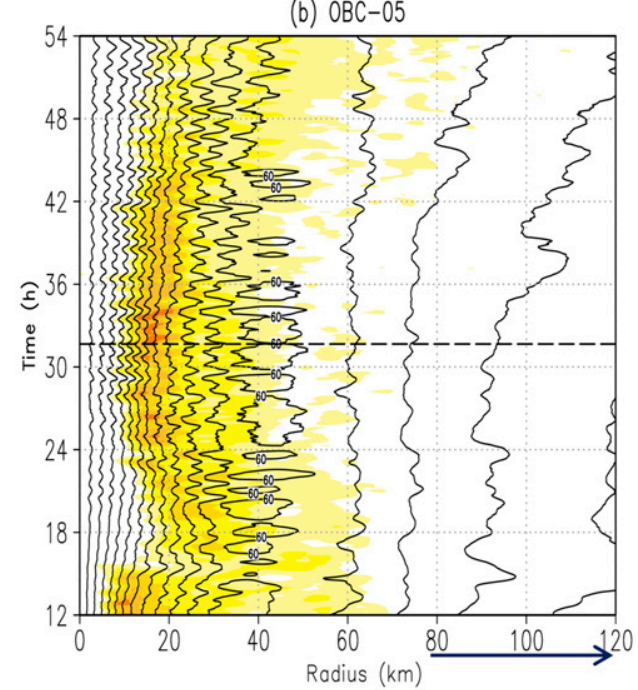

(d) $\mathrm{OBC}-15$

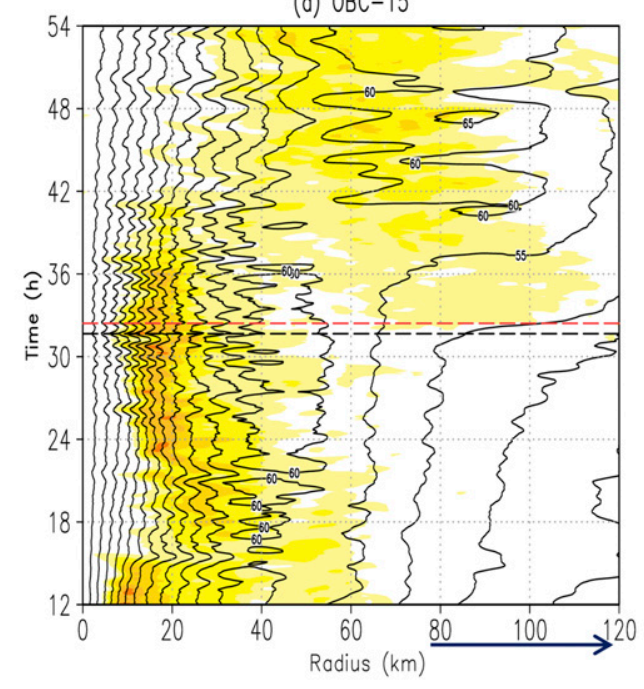

\section{$\begin{array}{llllllllll}0.2 & 0.4 & 0.6 & 0.8 & 1 & 1.2 & 1.4 & 1.6 & 2 & 2.4\end{array}$}

FIG. 8. As in Fig. 7, but for (a) OBC-01, (b) OBC-05, (c) OBC-10, and (d) OBC-15.

increase is due to the suppression of convection and the accompanying heating outside the primary eyewall. Note that the inertial stability increases in the SEF region (at radii between 50 and $80 \mathrm{~km}$ ), which cannot be distinguished from the primary eyewall since the moat is less clear during the second episode of SEF in RiR-10. In contrast, capping the WISHE feedback in the inner region does not greatly affect the establishment of an area of high inertial stability. In OSC-05, the tangential wind field expands with time (Fig. 7b), leading to the increased inertial stability outside the primary eyewall. Nevertheless, this is not enough to initiate SEF, which also requires more active convection and accompanying heating. In RiR-10, the convection expands with time, accompanied by expansion of the tangential wind field after $18 \mathrm{~h}$ (Fig. 9c). This expansion leads to one peak in the increase of inertial stability that extends from 40 - to $120-\mathrm{km}$ radii.

Figure 14 shows radius-height diagrams of variables relating to unbalanced dynamics in the lower troposphere around the original SEF time in CTL (OSC-05) and the SEF time of each experiment. The OSC-05 experiment, in which SEF is not observed, has only one dominant peak of supergradient winds with an outflow above the boundary layer. On the other hand, experiments with $\mathrm{SEF}$, that is, OSC-10, OSC-15, and InC-05, all have two distinct peaks in the supergradient wind. In OSC-10 and OSC-15, in which the WISHE feedback is capped around 
(o) RiR-01

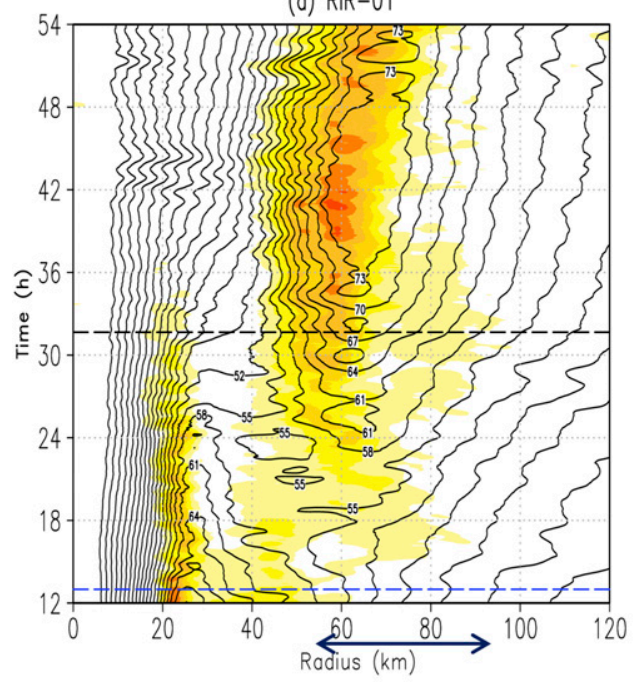

(c) RiR-10

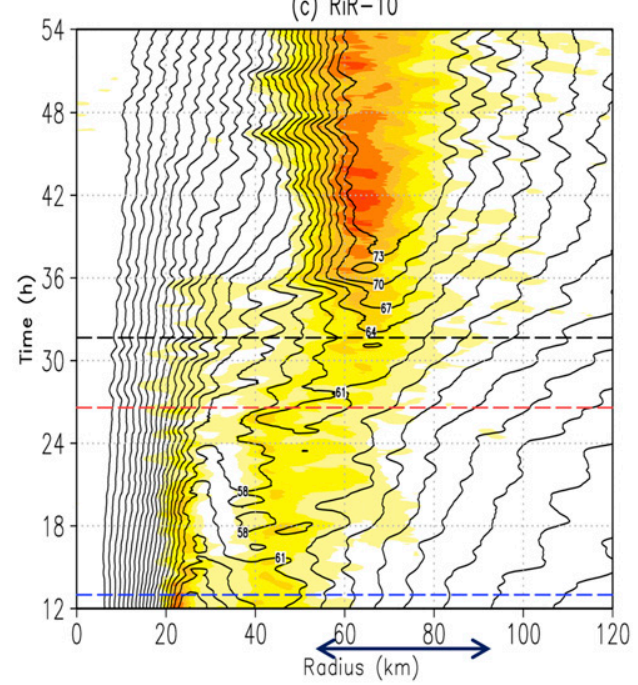

(b) RiR-05

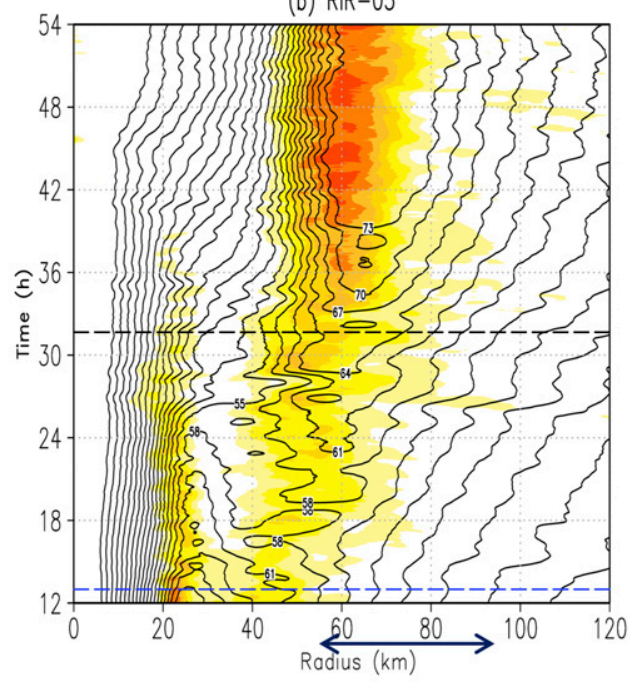

(d) RiR-15

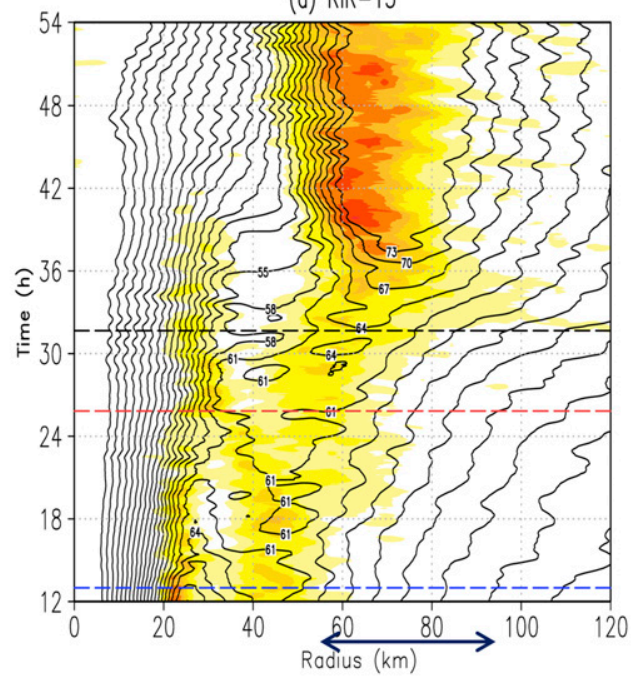

\section{$\begin{array}{llllllllll}0.2 & 0.4 & 0.6 & 0.8 & 1 & 1.2 & 1.4 & 1.6 & 2 & 2.4\end{array}$}

FIG. 9. As in Fig. 7, but for (a) RiR-01, (b) RiR-05, (c) RiR-10, and (d) RiR-15. The blue dashed line indicates the SEF time of the first episode of SEF, and the red dashed line indicates the second episode. The interval of the tangential wind in this figure is smaller than that in Fig. 7.

the SEF region, the secondary peak of supergradient flow and outflow atop the boundary layer is weaker than those in CTL (cf. Figs. 4c-e). Moreover, for RiR-10 and RiR-15, there exists a wide and continuous area of supergradient winds from 20 - to $120-\mathrm{km}$ radii. There are three unseparated peaks of supergradient winds in RiR-10, which is well correlated with the peaks of diabatic heating (eyewalls). In addition, the two peaks of supergradient winds in RiR-15 are consistent with the diabatic heating distribution of RiR-10 shown in Fig. 12e. The results in Fig. 14 show that variables related to unbalanced dynamics can be found in the experiments with SEF, even if the surface heat fluxes are capped. Suppressing the surface heat fluxes weakens the magnitude of the supergradient winds and the outflows at the top of the boundary layer near the secondary eyewall.

\section{Discussion and conclusions}

\section{a. Summary}

In this study, high-resolution numerical simulations of Typhoon Sinlaku (2008) are conducted to examine the role of WISHE in SEF. Results from CTL show that the 


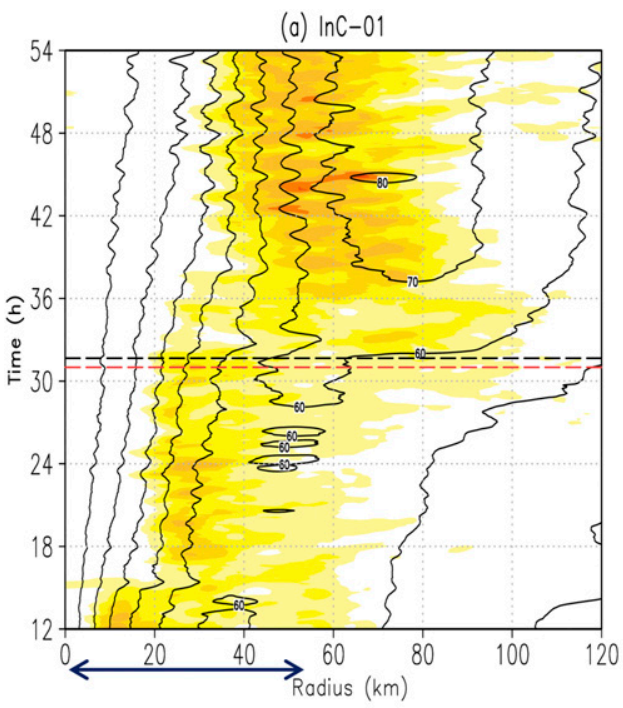

(c) $\ln \mathrm{C}-10$

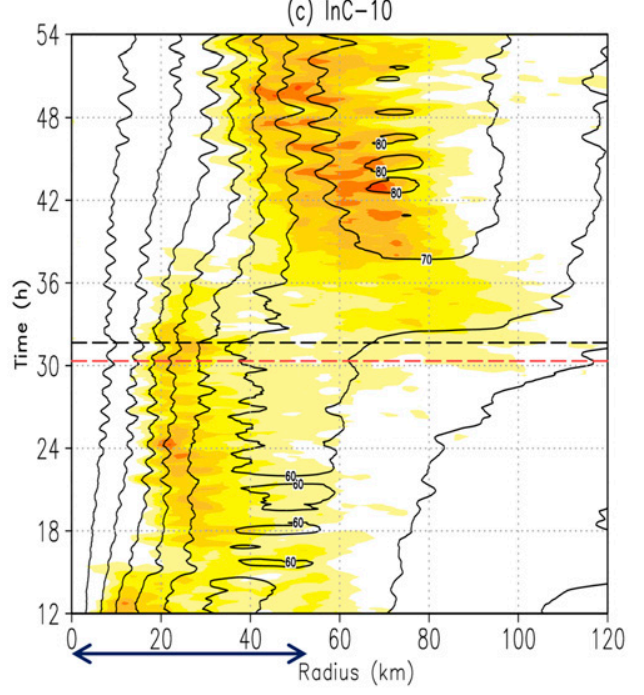

(b) $\ln C-05$

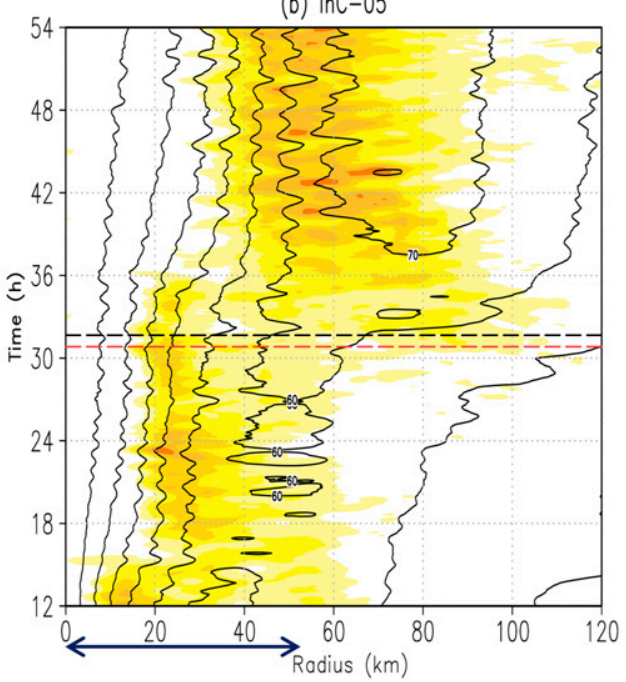

(d) $\ln \mathrm{C}-15$

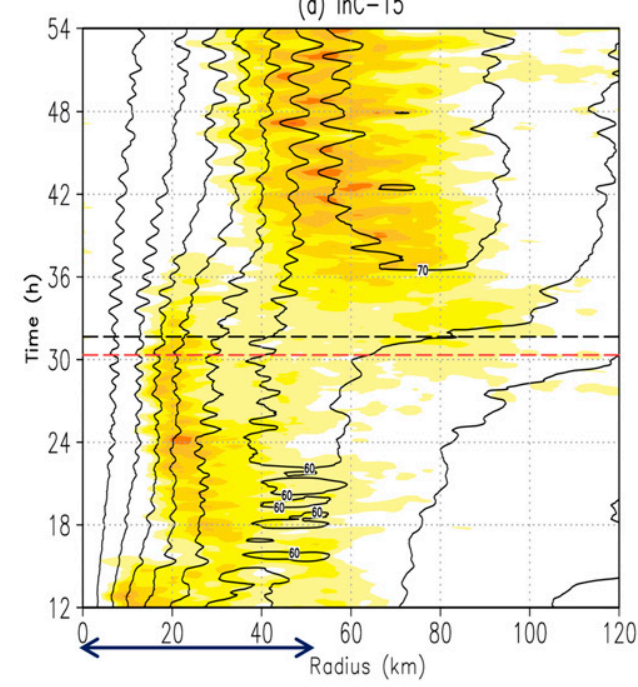

\section{$\begin{array}{llllllllll}0.2 & 0.4 & 0.6 & 0.8 & 1 & 1.2 & 1.4 & 1.6 & 2 & 2.4\end{array}$}

FIG. 10. As in Fig. 7, but for (a) InC-01, (b) InC-05, (c) InC-10, and (d) InC-15.

evolution of the vortex (e.g., secondary peaks of tangential wind, convection, and vertical velocity outside the primary eyewall) is consistent with previous observational and numerical studies (e.g., Bell et al. 2012; Wu et al. 2012; Huang et al. 2012; Rozoff et al. 2012). As the tangential wind field expands, the feedback between the surface winds and surface heat fluxes is shown to be an important process for the evolution of the outer eyewall and eventual storm intensity.

Analyses from the sensitivity experiments with suppressed WISHE feedback in different regions show that WISHE can affect the overall evolution and characteristics of the concentric eyewalls. When the heat fluxes are suppressed both around and outside the SEF region, the onset of the outer eyewall is delayed and the intensity of the eyewall is weaker in cases where WISHE is slightly or moderately capped (OSC-10, OSC-15, OBC-10, and OBC-15). When heat fluxes are strongly capped in the same region(s) (OSC-01, OSC-05, OBC-01, and OBC-05), SEF does not occur. Suppression of WISHE within the SEF area of CTL (the ring region), a relatively small area, can affect the structure of the storm as well. A different evolution of the inner-core structure is observed in the RiR experiments, which indicates that the primary eyewall is sensitive to the surface heat fluxes in the ring region. Artificially suppressing the surface heat 


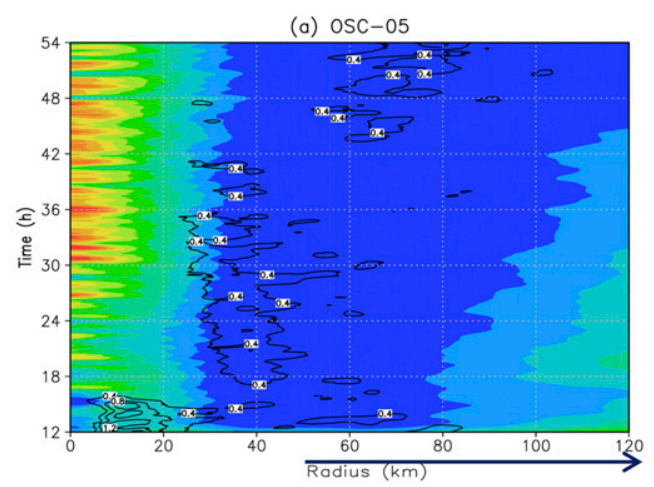

(c) $0 \mathrm{SC}-15$

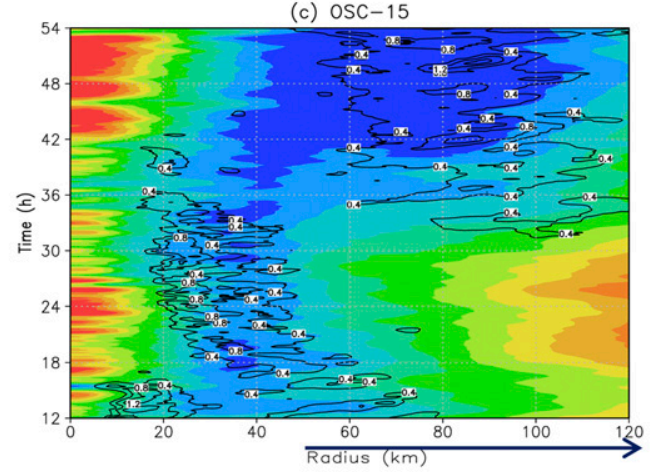

(e) RiR-10

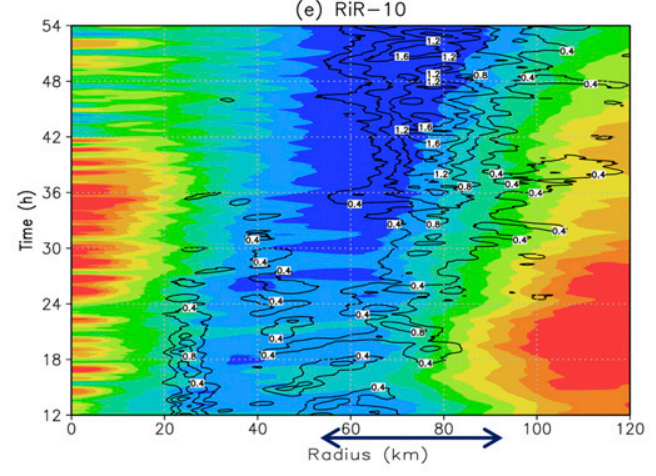

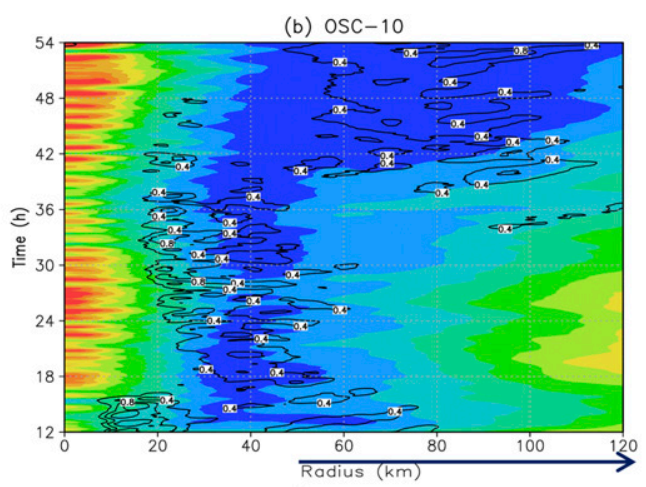

(d) $\ln \mathrm{C}-05$

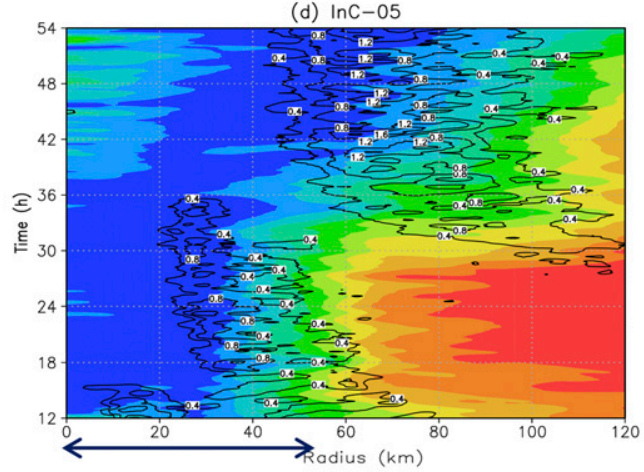

(f) RiR-15

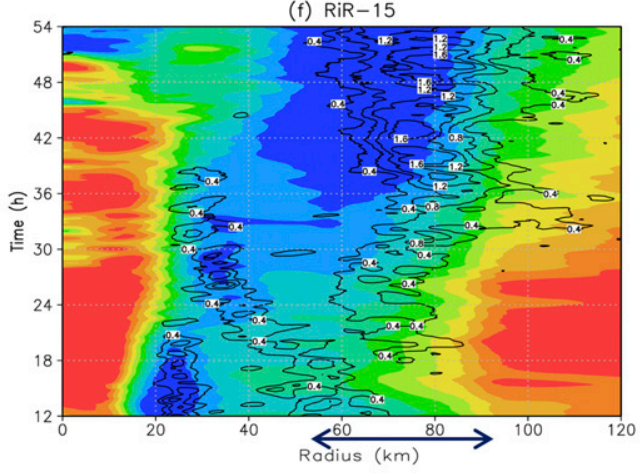

18002000

FIG. 11. Time-radius Hovmöller diagrams of the azimuthal-mean vertical velocity at 3-km altitude (contours for values $\geq 0.4 \mathrm{~m} \mathrm{~s}^{-1}$ ) and CAPE (shaded; $\mathrm{J} \mathrm{kg}^{-1}$ ) for (a) OSC-05, (b) OSC-10, (c) OSC-15, (d) InC-05, (e) RiR-10, and (f) RiR-15. The dark blue arrows indicate the capped area in each experiment.

fluxes limits the amount of heat energy fed into the primary eyewall, since the radial heat energy is carried by the inflow passing over the heat-flux-reduced region. In contrast, WISHE in the inner-core region (InC) is of limited importance in SEF, as demonstrated in the InC experiments.

Supergradient winds and the accompanying strengthening of convection in the SEF region are observed in all cases with SEF. On the contrary, these features are not found in the cases with neither SEF nor active convection outside the primary eyewall (i.e., OSC-01,
OSC-05, OBC-01, and OBC-05). Possible explanations involve both balanced and unbalanced dynamical processes, as well as their mutual interaction. The suppressed WISHE feedback creates less favorable environmental conditions for the development of convection, such as lower CAPE. The weaker overturning circulation in response to the reduced convection inhibits the expansion of the tangential wind field into the outer region. Correspondingly, the establishment of a region of enhanced inertial stability and supergradient winds outside the original eyewall is likely restricted. The former leads 


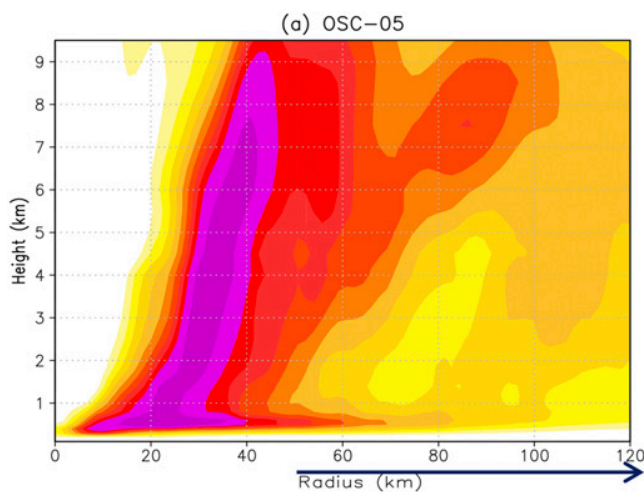

(c) $\mathrm{OSC}-15$

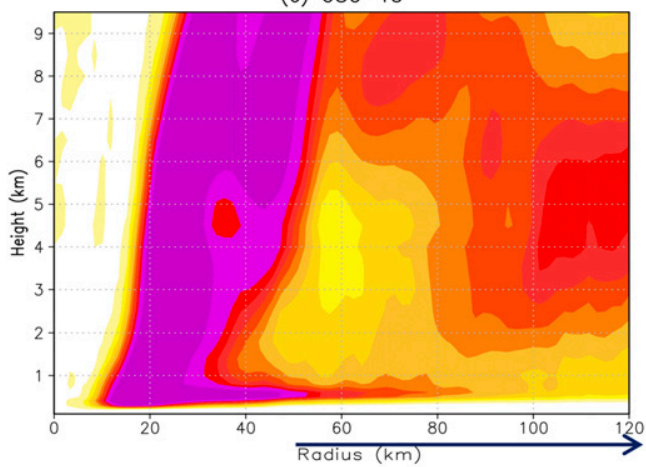

(e) RiR-10

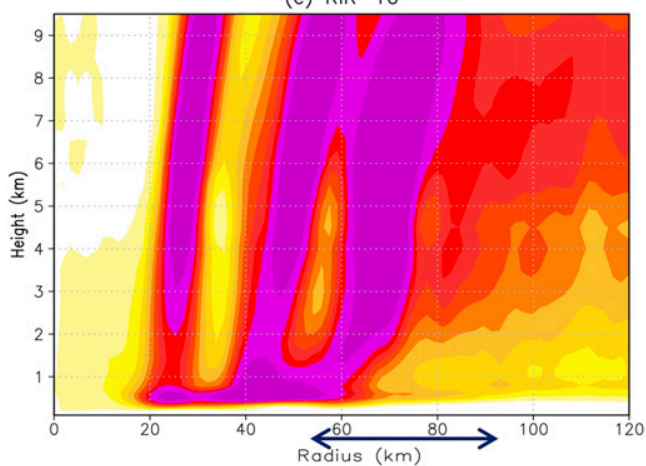

(b) $\mathrm{OSC}-10$

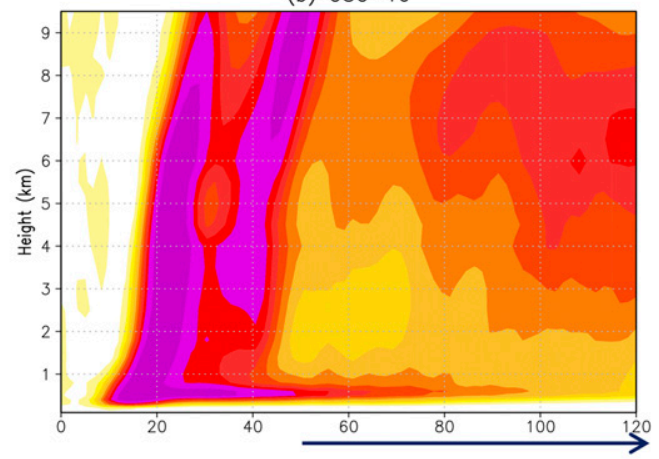

(d) $\ln \mathrm{C}-05$

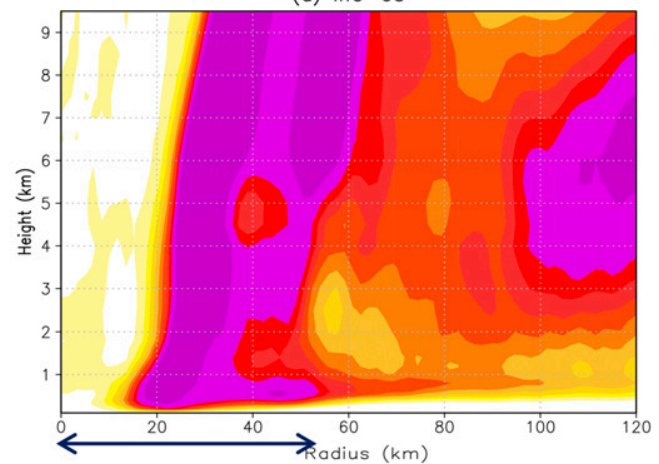

(f) RiR-15

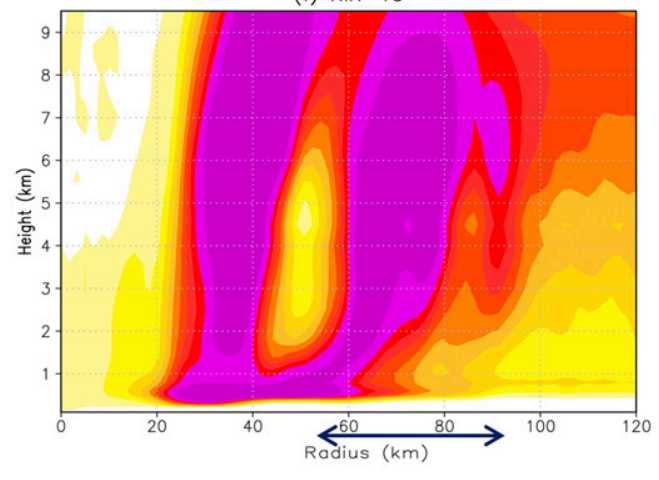

\section{0}

FIG. 12. Radius-height cross sections of the azimuthal-mean diabatic heating rate $\left(\mathrm{K} \mathrm{h}^{-1}\right)$. It is averaged from 27 to $32 \mathrm{~h}$ in (a) OSC-05 and averaged in the last $5 \mathrm{~h}$ before the time of SEF in (b) OSC-10, (c) OSC-15, (d) InC-05, (e) RiR-10, and (f) RiR-15 (for every 5-min output). The dark blue arrows indicate the capped area in each experiment.

to lower heating efficiency, and the latter results in weaker convergence in the boundary layer in the SEF region, stopping SEF from occurring.

\section{b. Discussion and comparison}

Previous studies (e.g., Montgomery et al. 2009, 2015; Zhang and Emanuel 2016) examined the role of WISHE in the intensification of TCs. The focus of this study is on SEF processes; however, the intensity evolution of the sensitivity experiments is similar to these studies. First, all of the previous studies showed that the intensification rate is reduced and the storm stabilizes at a weaker intensity when WISHE is limited. In this study, slightly or moderately suppressing the WISHE mechanism around and outside the SEF region (OSC-10, OSC-15, OBC-10, and OBC-15) leads to delayed SEF and a weaker outer eyewall (cf. Figs. 6c,d and Figs. 7c,d). In contrast, there are no SEF in experiments where 


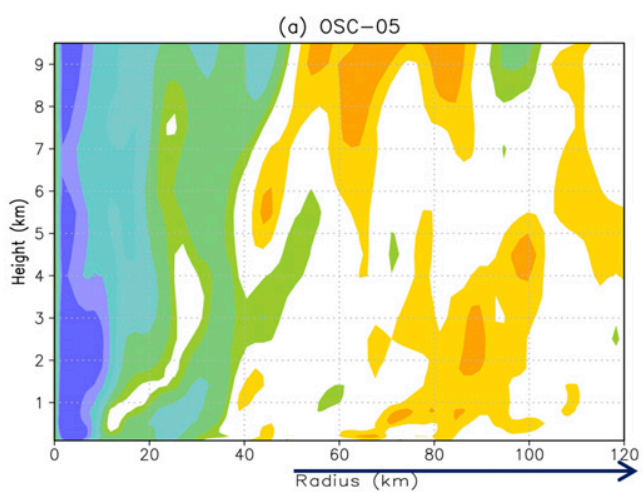

(c) OSC-15

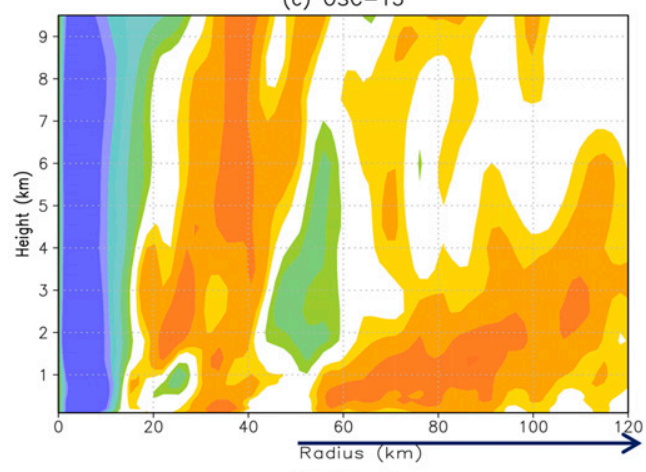

(e) RiR-10

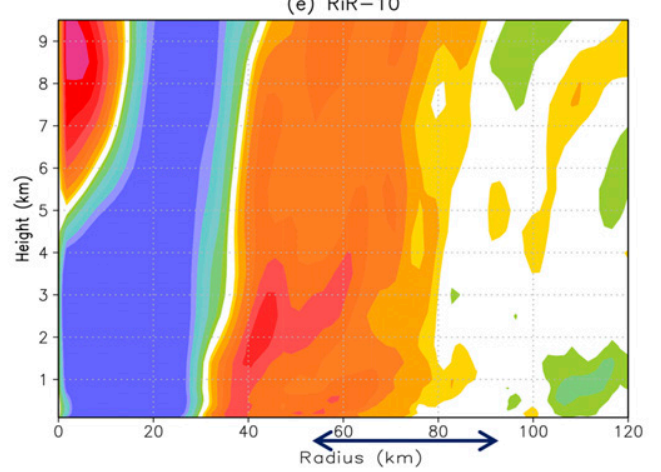

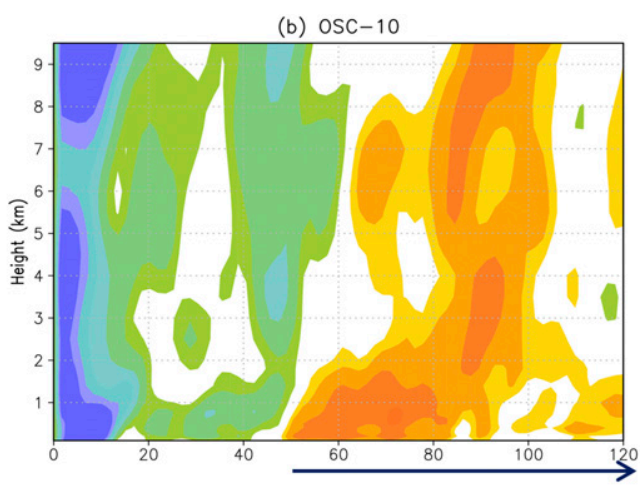

(d) $\ln \mathrm{C}-05$

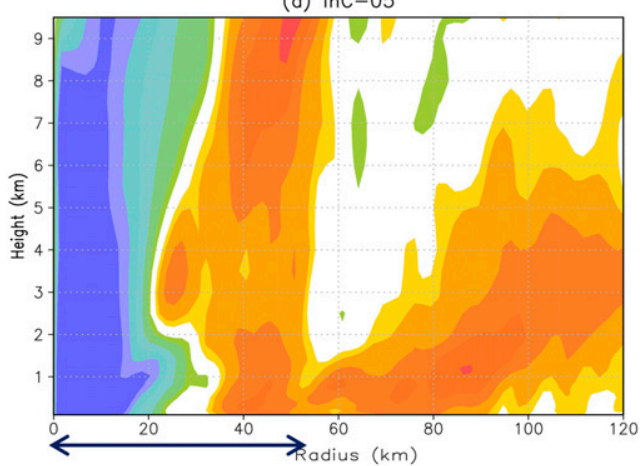

(f) RiR-15

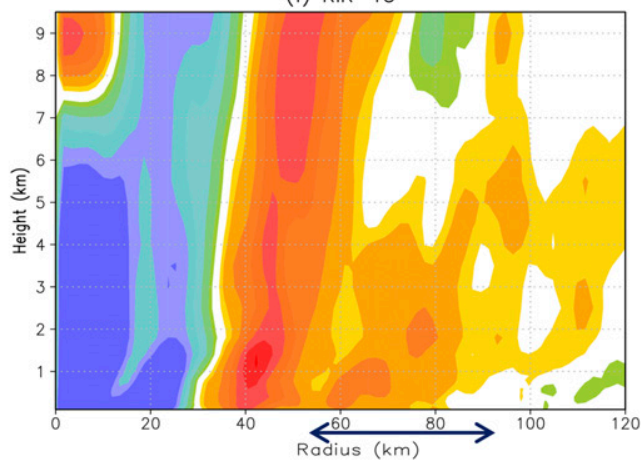

$\begin{array}{llllllllllll}-0.5 & -0.4-0.3-0.2-0.1-0.050 .05 & 0.1 & 0.2 & 0.3 & 0.4 & 0.5 & 0.6 & 0.7 & 0.8 & 1\end{array}$

FIG. 13. Radius-height cross sections of the changes in the azimuthal-mean inertial stability $\left(\mathrm{s}^{-1}\right)$ between 22 and $32 \mathrm{~h}$ in (a) OSC-05, and the change in the last $10 \mathrm{~h}$ before the time of SEF in (b) OSC-10, (c) OSC-15, (d) InC-05, (e) RiR-10, and (f) RiR-15. The dark blue arrows indicate the capped area in each experiment.

WISHE is strongly suppressed around and outside the outer eyewall (OSC-01, OSC-05, OBC-01, and OBC-05). Such a result can be connected to that of Zhang and Emanuel (2016) with regard to the case of Hurricane Edouard (2014), in which there was little intensification when the wind speed used for calculating fluxes was capped at $5 \mathrm{~m} \mathrm{~s}^{-1}$.

Meanwhile, Xu and Wang (2010) pointed out that the surface entropy fluxes outside the inner core play a critical role in maintaining high CAPE outside the eyewall and spiral rainbands and are thus important for the increase in the inner-core size. Results of this study are consistent with those of Xu and Wang (2010) that the WISHE mechanism (mechanisms related to surface entropy fluxes) outside the outer eyewall is important for its development and that SEF processes can lead to an increase in the inner-core size. This increase in the inner-core size is evident in the expansion of the tangential wind field (Fig. 2a) and increasing PV in the radial direction (Fig. 3c).

Simulations in this study are based on a real case, the results of which could be affected by some environmental factors, such as the asymmetric processes 


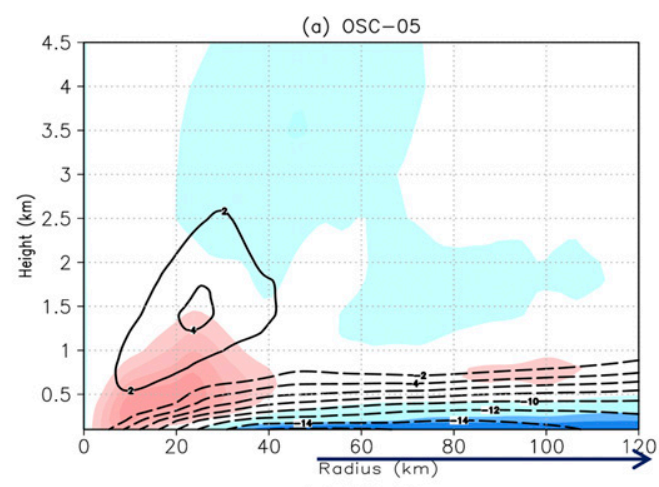

(c) $\mathrm{OSC}-15$

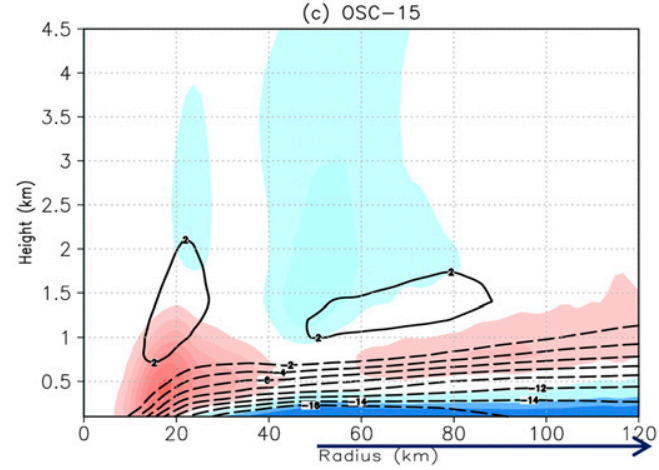

(e) RiR-10

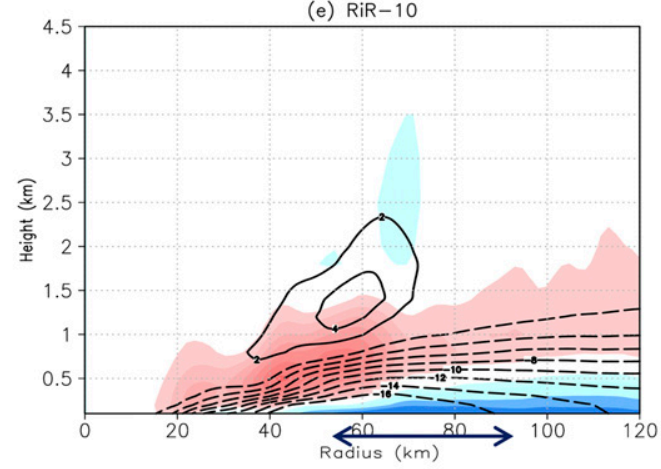

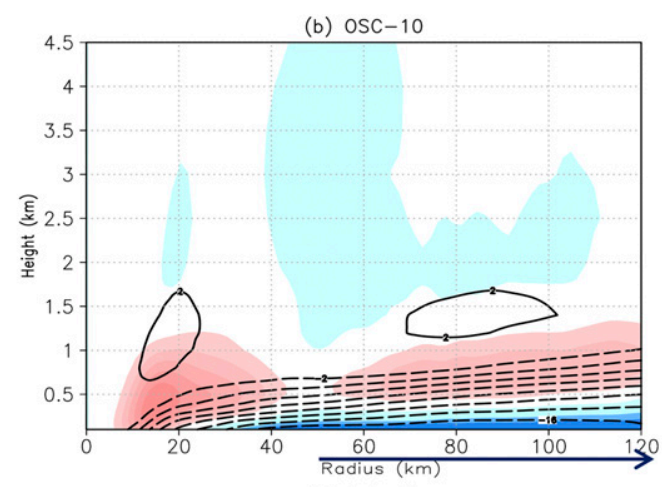

(d) $\operatorname{InC}-05$

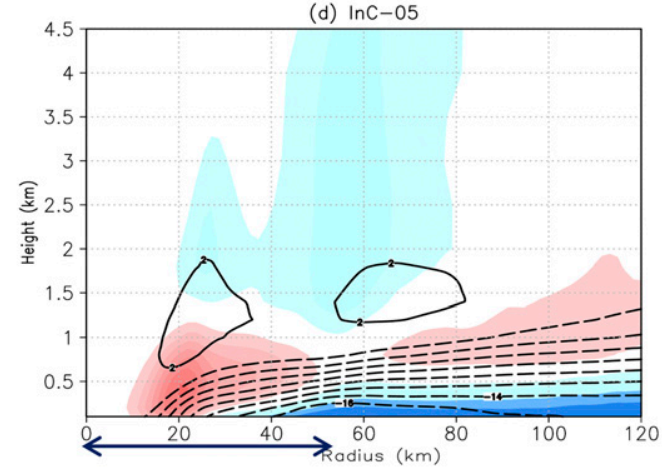

(f) $R$ RiR-15

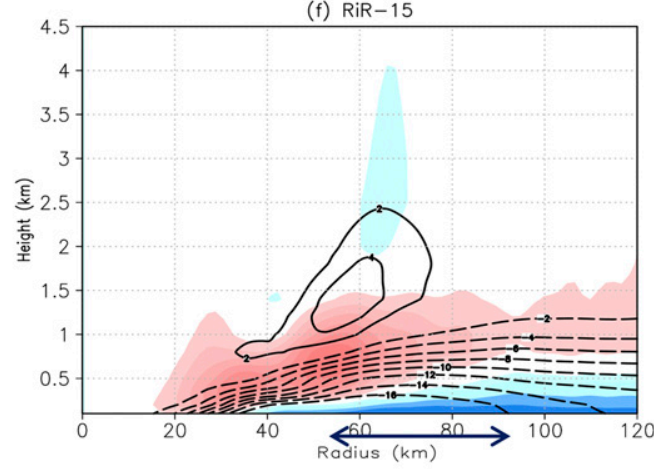

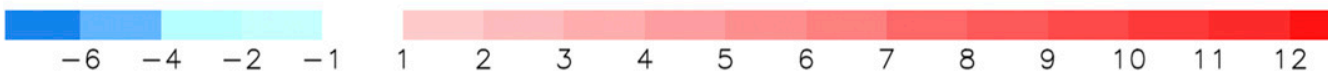

FIG. 14. Radius-height diagrams of the subgradient wind (blue shaded; $\mathrm{m} \mathrm{s}^{-1}$ ), supergradient wind (red shaded; $\mathrm{m} \mathrm{s}^{-1}$ ), radial inflow (black dashed lines; $\mathrm{m} \mathrm{s}^{-1}$ ), and radial outflow (black solid lines; $\mathrm{m} \mathrm{s}^{-1}$ ). These parameters are averaged from 27 to $32 \mathrm{~h}$ in (a) OSC-05 and averaged in the last $5 \mathrm{~h}$ before SEF in (b) OSC-10, (c) OSC-15, (d) InC-05, (e) RiR-10, and (f) RiR-15. The dark blue arrows indicate the capped area in each experiment.

associated with vertical wind shear. Therefore, idealized simulations need to be conducted to further examine the role of WISHE in SEF. Ensemble simulations could also be used to understand the uncertainty associated with these SEF- and WISHE-related processes.

Acknowledgments. This work is supported by the Ministry of Science and Technology of Taiwan under Grants MOST 105-2628-M-002-001 and MOST 106-2111M-002-013-MY3. Valuable comments from Yi-Hsuan
Huang, the editor, and three anonymous reviewers that helped improve the quality of the manuscript are highly appreciated.

\section{REFERENCES}

Abarca, S. F., and M. T. Montgomery, 2013: Essential dynamics of secondary eyewall formation. J. Atmos. Sci., 70, 3216-3230, https://doi.org/10.1175/JAS-D-12-0318.1.

American Meteorological Society, 2013: Wind-induced surface heat exchange. Glossary of Meteorology, http://glossary. ametsoc.org/wiki/Wind-induced_surface_heat_exchange. 
Bell, M. M., M. T. Montgomery, and W.-C. Lee, 2012: An axisymmetric view of concentric eyewall evolution in Hurricane Rita (2005). J. Atmos. Sci., 69, 2414-2432, https://doi.org/10.1175/ JAS-D-11-0167.1.

Didlake, A. C., and R. A. Houze, 2011: Kinematics of the secondary eyewall observed in Hurricane Rita (2005). J. Atmos. Sci., 68, 1620-1636, https://doi.org/10.1175/2011JAS3715.1.

Dudhia, J., 1989: Numerical study of convection observed during the Winter Monsoon Experiment using a mesoscale twodimensional model. J. Atmos. Sci., 46, 3077-3107, https:// doi.org/10.1175/1520-0469(1989)046<3077:NSOCOD>2.0.CO;2.

Eliassen, A., 1951: Slow thermally or frictionally controlled meridional circulation in a circular vortex. Astrophys. Norv., 5, 19-60.

Emanuel, K. A., 1986: An air-sea interaction theory for tropical cyclones. Part I: Steady-state maintenance. J. Atmos. Sci., 43, 585-605, https://doi.org/10.1175/1520-0469(1986)043<0585: AASITF $>2.0 . \mathrm{CO} ; 2$.

__ 1989: The finite-amplitude nature of tropical cyclogenesis. J. Atmos. Sci., 46, 3431-3456, https://doi.org/10.1175/ 1520-0469(1989)046<3431:TFANOT>2.0.CO;2.

- 1997: Some aspects of hurricane inner-core dynamics and energetics. J. Atmos. Sci., 54, 1014-1026, https://doi.org/10.1175/ 1520-0469(1997)054<1014:SAOHIC >2.0.CO;2.

Hawkins, J. D., and M. Helveston, 2008: Tropical cyclone multiple eyewall characteristics. 28th Conf. Hurricanes and Tropical Meteorology, Orlando, FL, Amer. Meteor. Soc., 14B.1, https://ams. confex.com/ams/28Hurricanes/techprogram/paper_138300.htm.

Hong, S.-Y., and J.-O. J. Lim, 2006: The WRF single-moment 6-class microphysics scheme (WSM6). J. Korean Meteor. Soc., 42, 129-151.

_- Y. Noh, and J. Dudhia, 2006: A new vertical diffusion package with an explicit treatment of entrainment processes. Mon. Wea. Rev., 134, 2318-2341, https://doi.org/10.1175/MWR3199.1.

Houze, R. A., Jr., and Coauthors, 2006: The Hurricane Rainband and Intensity Change Experiment: Observations and modeling of Hurricanes Katrina, Ophelia, and Rita. Bull. Amer. Meteor. Soc., 87, 1503-1521, https://doi.org/10.1175/BAMS-87-11-1503.

- S. S. Chen, B. F. Smull, W.-C. Lee, and M. M. Bell, 2007: Hurricane intensity and eyewall replacement. Science, 315, 1235-1239, https://doi.org/10.1126/science.1135650.

Huang, Y.-H., M. T. Montgomery, and C.-C. Wu, 2012: Concentric eyewall formation in Typhoon Sinlaku (2008). Part II: Axisymmetric dynamical processes. J. Atmos. Sci., 69, 662-674, https://doi.org/10.1175/JAS-D-11-0114.1.

Jiménez, P. A., J. Dudhia, J. F. González-Rouco, J. Navarro, J. P. Montávez, and E. García-Bustamante, 2012: A revised scheme for the WRF surface layer formulation. Mon. Wea. Rev., 140, 898-918, https://doi.org/10.1175/MWR-D-11-00056.1.

Kain, J. S., and J. M. Fritsch, 1993: Convective parameterization for mesoscale models: The Kain-Fritsch scheme. The Representation of Cumulus Convection in Numerical Models, Meteor. Monogr., No. 46, Amer. Meteor. Soc., 165-177.

Kepert, J. D., 2013: How does the boundary layer contribute to eyewall replacement cycles in axisymmetric tropical cyclones? J. Atmos. Sci. 70, 2808-2830, https://doi.org/10.1175/JAS-D-13-046.1.

— the boundary layer contribute to eyewall replacement cycles in axisymmetric tropical cyclones?"' J. Atmos. Sci., 71, 46924704, https://doi.org/10.1175/JAS-D-14-0014.1.

Kleinschmidt, E., Jr., 1951: Grundlagen einer theorie der tropischen zyklonen. Arch. Meteor. Geophys. Bioklimatol., 4A, 53-72, https://doi.org/10.1007/BF02246793.
Kossin, J. P., and M. Sitkowski, 2009: An objective model for identifying secondary eyewall formation in hurricanes. Mon. Wea. Rev., 137, 876-892, https://doi.org/10.1175/2008MWR2701.1.

Kuo, H.-C., C.-P. Chang, Y.-T. Yang, and H.-J. Jiang, 2009: Western North Pacific typhoons with concentric eyewalls. Mon. Wea. Rev., 137, 3758-3770, https://doi.org/10.1175/2009MWR2850.1.

Mlawer, E. J., S. J. Taubman, P. D. Brown, M. J. Iacono, and S. A. Clough, 1997: Radiative transfer for inhomogeneous atmosphere: RRTM, a validated correlated- $k$ model for the longwave. J. Geophys. Res., 102, 16663-16682, https://doi.org/ 10.1029/97JD00237.

Montgomery, M. T., N. V. Sang, R. K. Smith, and J. Persing, 2009: Do tropical cyclones intensify by WISHE? Quart. J. Roy. Meteor. Soc., 135, 1697-1714, https://doi.org/10.1002/qj.459.

—, S. F. Abarca, R. K. Smith, C.-C. Wu, and Y.-H. Huang, 2014: Comments on "How does the boundary layer contribute to eyewall replacement cycles in axisymmetric tropical cyclones?" J. Atmos. Sci., 71, 4682-4691, https://doi.org/10.1175/JAS-D-13-0286.1.

__ J. Jersing, and R. K. Smith, 2015: Putting to rest WISHEful misconceptions for tropical cyclone intensification. J. Adv. Model. Earth Syst., 7, 92-109, https://doi.org/10.1002/ 2014MS000362.

Ooyama, K., 1969: Numerical simulation of the life cycle of tropical cyclones. J. Atmos. Sci., 26, 3-40, https://doi.org/ 10.1175/1520-0469(1969)026<0003:NSOTLC $>2.0$.CO;2.

Riehl, H., 1950: A model for hurricane formation. J. Appl. Phys., 21, 917-925, https://doi.org/10.1063/1.1699784.

Rotunno, R., and K. A. Emanuel, 1987: An air-sea interaction theory for tropical cyclones. Part II: Evolutionary study using a nonhydrostatic axisymmetric numerical model. J. Atmos. Sci., 44, 542-561, https://doi.org/10.1175/1520-0469(1987)044<0542: AAITFT $>2.0 . \mathrm{CO} ; 2$.

Rozoff, C. M., D. S. Nolan, J. P. Kossin, F. Zhang, and J. Fang, 2012: The roles of an expanding wind field and inertial stability in tropical cyclone secondary eyewall formation. J. Atmos. Sci., 69, 2621-2643, https://doi.org/10.1175/JAS-D-11-0326.1.

Schubert, W. H., and J. J. Hack, 1982: Inertial stability and tropical cyclone development. J. Atmos. Sci., 39, 1687-1697, https://doi.org/ 10.1175/1520-0469(1982)039<1687:ISATCD>2.0.CO;2.

Shapiro, L. J., and H. E. Willoughby, 1982: The response of balanced hurricanes to local sources of heat and momentum. J. Atmos. Sci., 39, 378-394, https://doi.org/10.1175/1520-0469 (1982)039<0378:TROBHT>2.0.CO;2.

Sitkowski, M., J. P. Kossin, and C. M. Rozoff, 2011: Intensity and structure changes during hurricane eyewall replacement cycles. Mon. Wea. Rev., 139, 3829-3847, https://doi.org/10.1175/ MWR-D-11-00034.1.

Terwey, W. D., and M. T. Montgomery, 2008: Secondary eyewall formation in two idealized, full-physics modeled hurricanes. J. Geophys. Res., 113, D12112, https://doi.org/10.1029/ 2007JD008897.

Wang, H., C. Wu, and Y. Wang, 2016: Secondary eyewall formation in an idealized tropical cyclone simulation: Balanced and unbalanced dynamics. J. Atmos. Sci., 73, 3911-3930, https://doi.org/ 10.1175/JAS-D-15-0146.1.

Wang, X., Y. Ma, and N. E. Davidson, 2013: Secondary eyewall formation and eyewall replacement cycles in a simulated hurricane: Effect of the net radial force in the hurricane boundary layer. J. Atmos. Sci., 70, 1317-1341, https://doi.org/ 10.1175/JAS-D-12-017.1.

Wang, Y., 2009: How do outer spiral rainbands affect tropical cyclone structure and intensity? J. Atmos. Sci., 66, 1250-1273, https://doi.org/10.1175/2008JAS2737.1. 
Wu, C.-C., G.-Y. Lien, J.-H. Chen, and F. Zhang, 2010: A new approach for tropical cyclone initialization based on the ensemble Kalman filter (EnKF). J. Atmos. Sci., 67, 3806-3822, https://doi.org/10.1175/2010JAS3444.1.

_ , Y.-H. Huang, and G.-Y. Lien, 2012: Concentric eyewall formation in Typhoon Sinlaku (2008). Part I: Assimilation of T-PARC data based on the ensemble Kalman filter (EnKF). Mon. Wea. Rev., 140, 506-527, https://doi.org/10.1175/MWR-D-11-00057.1.
Xu, J., and Y. Wang, 2010: Sensitivity of tropical cyclone inner-core size and intensity to the radial distribution of surface entropy flux. J. Atmos. Sci., 67, 1831-1852, https://doi.org/10.1175/ 2010JAS3387.1.

Zhang, F., and K. A. Emanuel, 2016: On the role of surface fluxes and WISHE in tropical cyclone intensification. J. Atmos. Sci., 73, 2011-2019, https://doi.org/10.1175/JAS-D16-0011.1. 